\title{
Structural and thermal studies of new triphenyltin
}

\author{
complexes
}

Agata Owczarzakª, Maciej Kubickia, Anita M. Grześkiewicza*

aDepartment of Chemistry, Adam Mickiewicz University, Umultowska 89b, 61-614

Poznań, Poland

e-mail:aniow@amu.edu.pl

KEYWORDS: Organotin complexes, structural, thermal studies, triphenyltin, thioamides

Supplementary materials 
Table S1. Chemical structures of studied compounds

\section{Chemical structures of studied compounds}






\section{Ellipsoidal view if the structure together with labeling scheme}
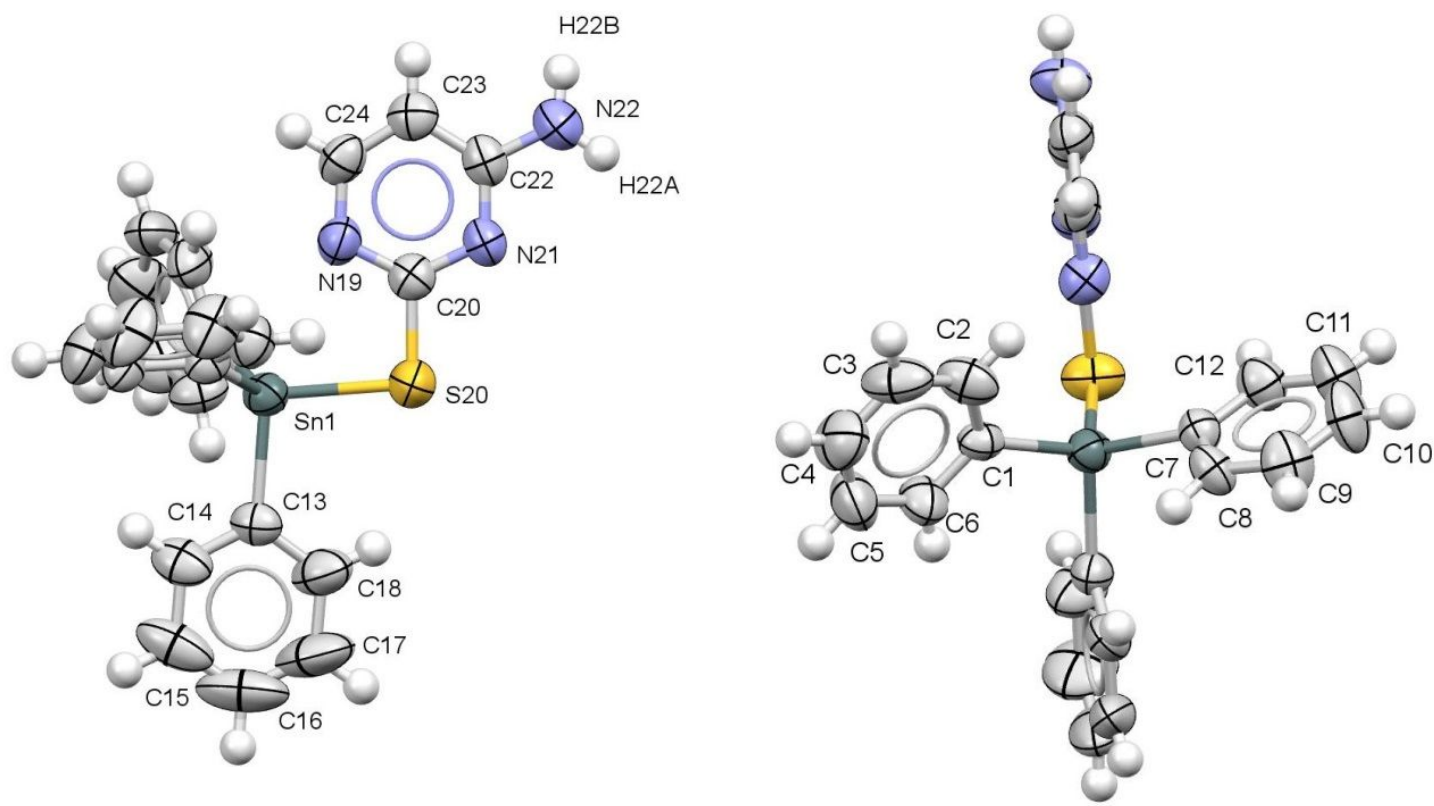

Figure S1.1 A perspective view of one of the complex $\mathbf{P h}_{3} \mathbf{S n} \_$TC (1) with the numbering scheme, ellipsoids are drawn at the $50 \%$ probability level, hydrogen atoms are shown as fixed-size spheres of radius $0.3 \AA$
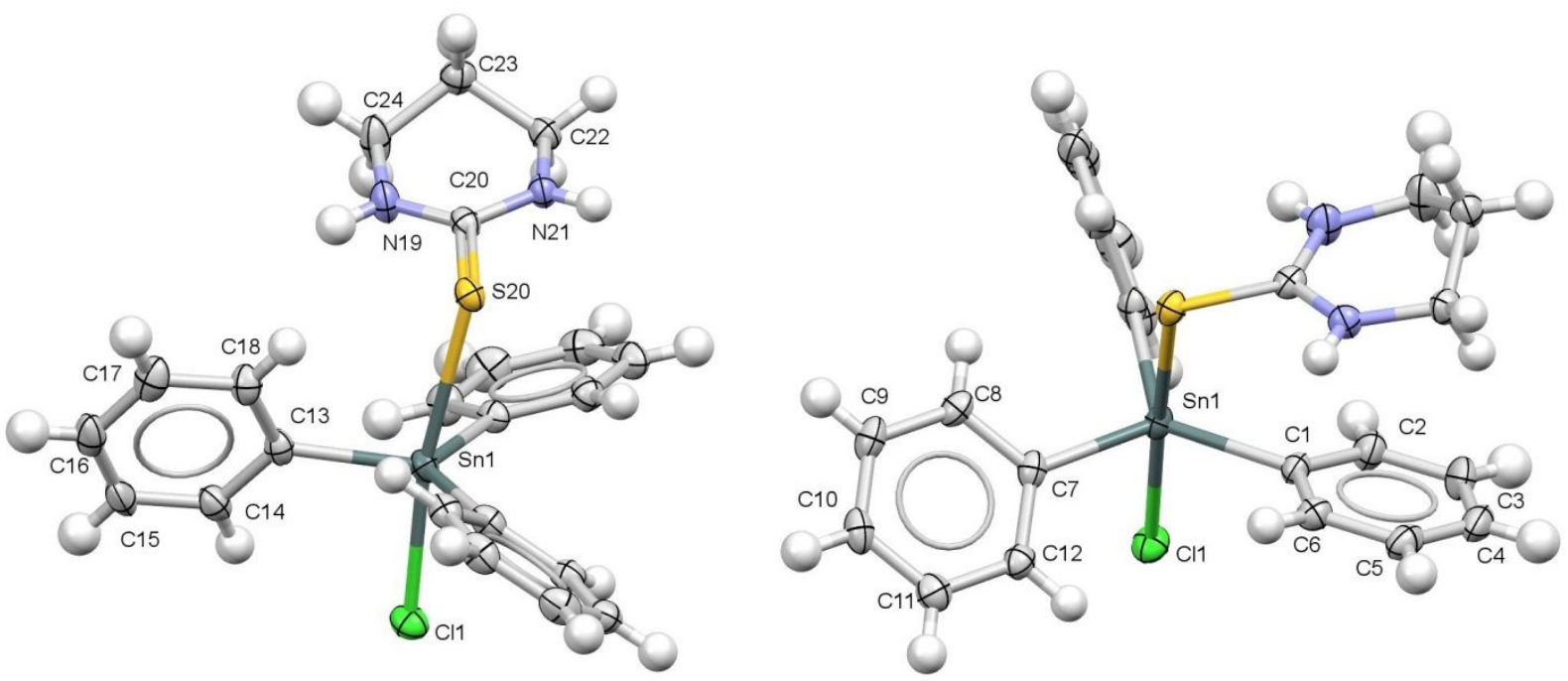

Figure S1.2 A perspective view of the complex Ph ${ }_{3}$ Sn_tHPMT_Cl (2) with the numbering scheme, ellipsoids are drawn at the $50 \%$ probability level, hydrogen atoms are shown as spheres of arbitrary radii. 

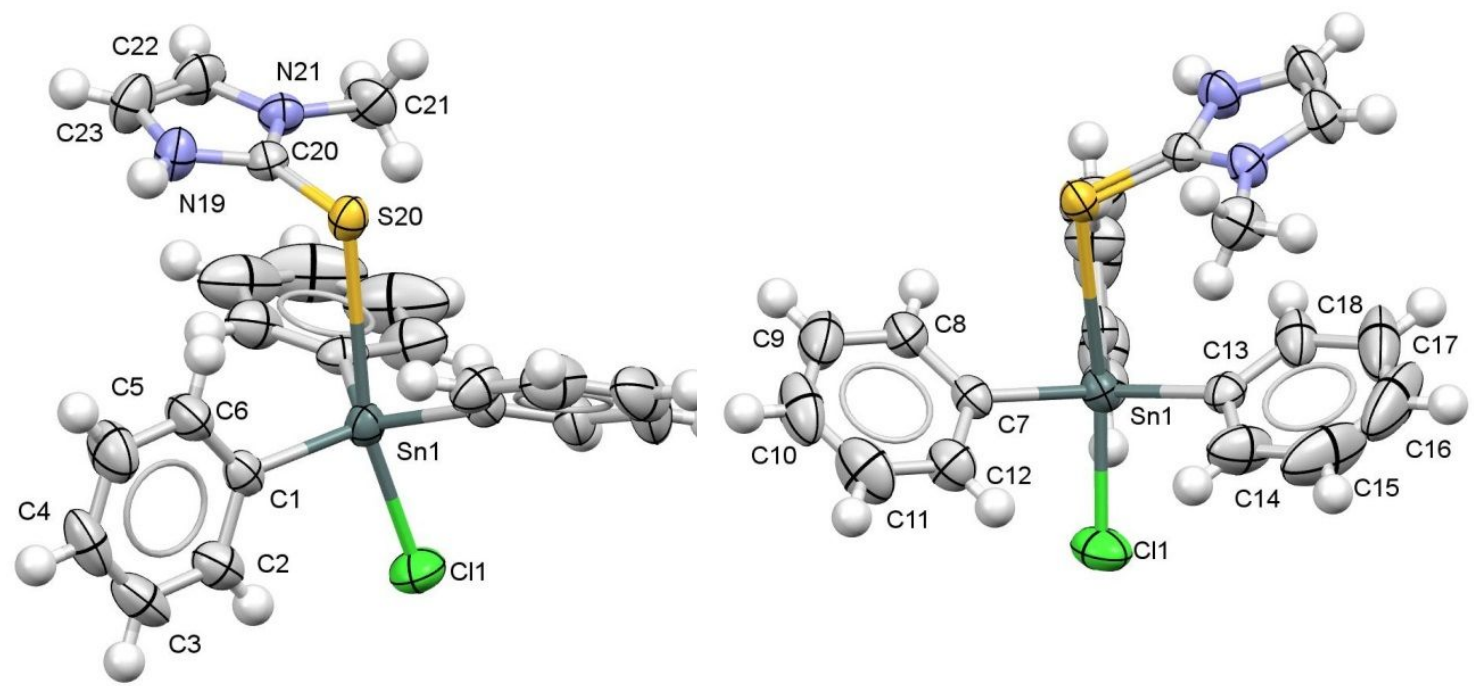

Figure S1.3a A perspective view of the complex $\mathbf{P h}_{3} \mathbf{S n}$ _MIMT_Cl (3a) with the numbering scheme, ellipsoids are drawn at the $50 \%$ probability level, hydrogen atoms are shown as spheres of arbitrary radii.
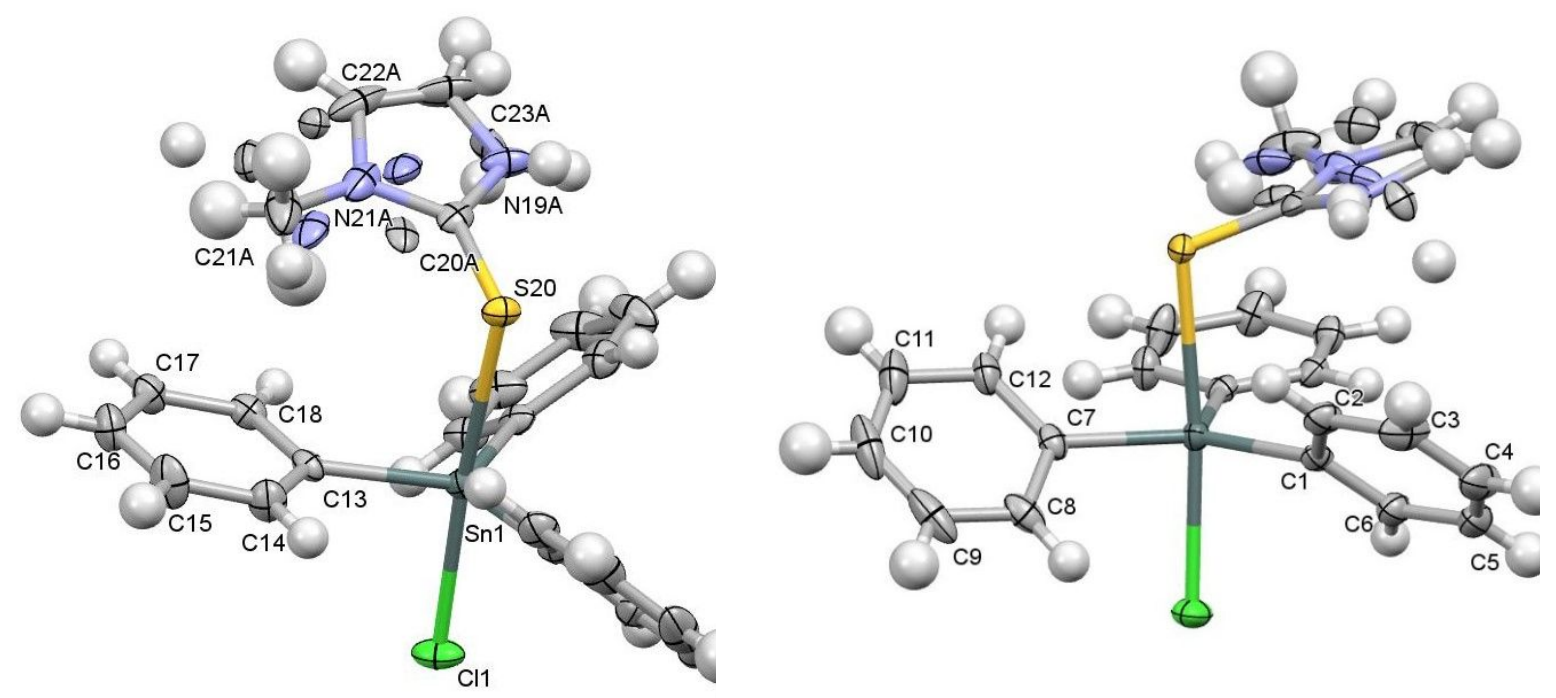

Figure S1.3b A perspective view of the complex $\mathbf{P h}_{3} \mathbf{S n}$ _MIMT_Cl (3a) with the numbering scheme, ellipsoids are drawn at the $50 \%$ probability level, hydrogen atoms are shown as spheres of arbitrary radii. 



Figure S1.4 A perspective view of the complex ) $\mathbf{P h}_{3} \mathbf{S n}_{-}(\mathbf{M I M T})_{2} . \mathbf{P h}_{3} \mathbf{S n C l}_{2}(4)$ with the numbering scheme, ellipsoids are drawn at the $50 \%$ probability level, hydrogen atoms are shown as spheres of arbitrary radii.

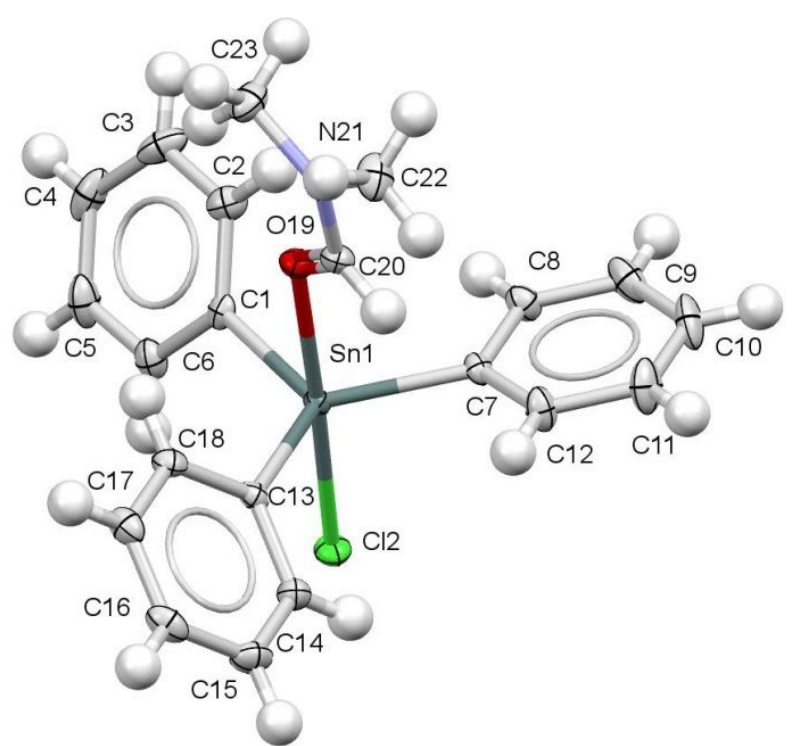

Figure S1.5 A perspective view of the complex $\mathrm{SnPh}_{3} \mathrm{Cl}_{-} \mathrm{DMF}(5)$ with the numbering scheme, ellipsoids are drawn at the $50 \%$ probability level, hydrogen atoms are shown as spheres of arbitrary radii. 

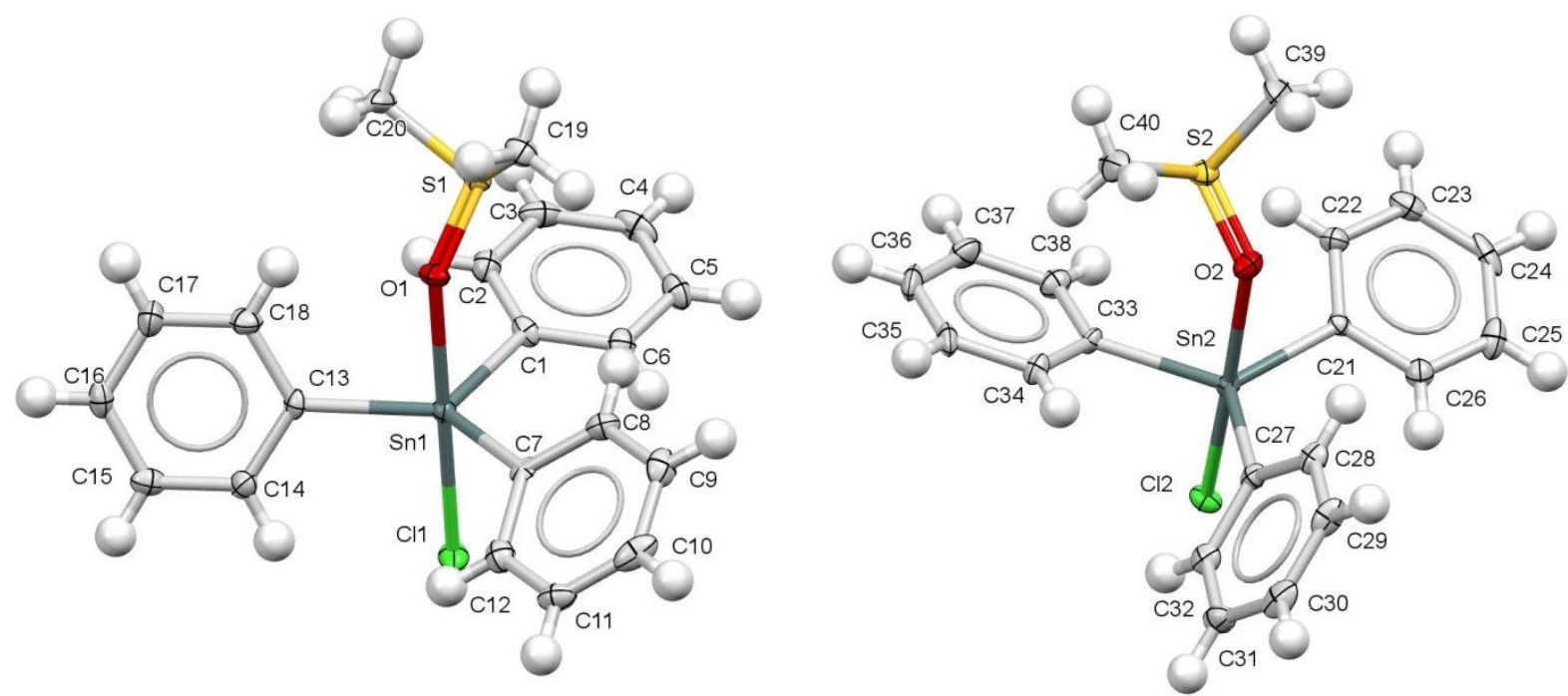

Figure S1.6 A perspective view of the complex $\mathbf{P h}_{3} \mathbf{S n}$ _DMSO_Cl (6a) with the numbering scheme, ellipsoids are drawn at the $50 \%$ probability level, hydrogen atoms are shown as spheres of arbitrary radii.
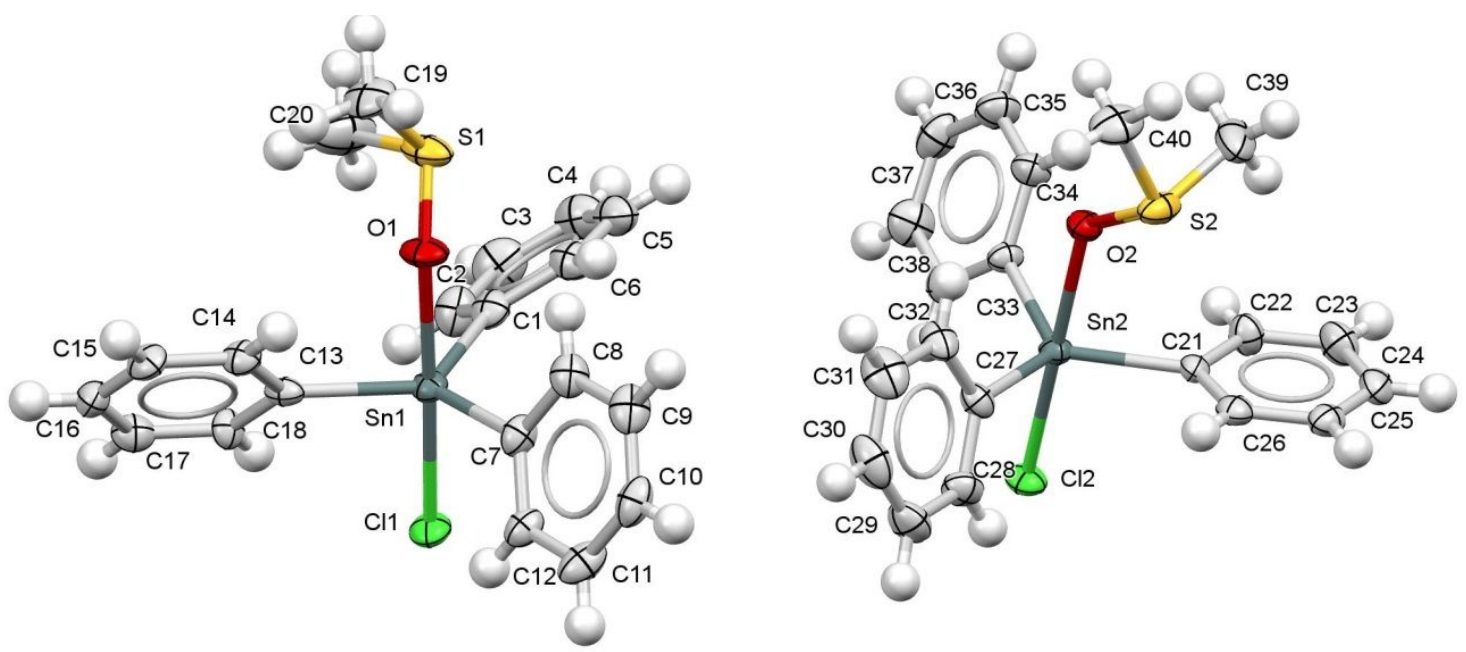

Figure S1.7 A perspective view of the complex $\mathbf{P h}_{3}$ Sn_DMSO_Cl (6b) with the numbering scheme, ellipsoids are drawn at the $50 \%$ probability level, hydrogen atoms are shown as spheres of arbitrary radii.

\section{Selected geometric parameters ( $\AA$, ㅇ)}

Table S2. Selected geometric parameters for all studied compounds

\section{$\mathrm{Ph}_{3} \mathrm{Sn} \_\mathrm{TC}(1)$}

$\begin{array}{llll}\mathrm{Sn} 1-\mathrm{C} 1 & 2.116(5) & \mathrm{C} 5-\mathrm{C} 6 & 1.380(7) \\ \mathrm{Sn} 1-\mathrm{C} 7 & 2.129(4) & \mathrm{C} 7-\mathrm{C} 8 & 1.375(6) \\ \mathrm{Sn} 1-\mathrm{C} 13 & 2.141(5) & \mathrm{C} 7-\mathrm{C} 12 & 1.377(6) \\ \mathrm{Sn} 1-\mathrm{S} 20 & 2.4280(13) & \mathrm{C} 8-\mathrm{C} 9 & 1.398(6) \\ \mathrm{S} 20-\mathrm{C} 20 & 1.750(5) & \mathrm{C} 9-\mathrm{C} 10 & 1.354(7) \\ \mathrm{N} 19-\mathrm{C} 20 & 1.329(5) & \mathrm{C} 10-\mathrm{C} 11 & 1.354(6)\end{array}$




\begin{tabular}{|c|c|c|c|}
\hline $\mathrm{N} 19-\mathrm{C} 24$ & $1.334(5)$ & $\mathrm{C} 11-\mathrm{C} 12$ & $1.396(6)$ \\
\hline $\mathrm{N} 21-\mathrm{C} 20$ & $1.327(5)$ & $\mathrm{C} 13-\mathrm{C} 18$ & $1.369(6)$ \\
\hline $\mathrm{N} 21-\mathrm{C} 22$ & $1.336(6)$ & $\mathrm{C} 13-\mathrm{C} 14$ & $1.376(6)$ \\
\hline $\mathrm{N} 22-\mathrm{C} 22$ & $1.342(5)$ & $\mathrm{C} 14-\mathrm{C} 15$ & $1.374(7)$ \\
\hline $\mathrm{C} 1-\mathrm{C} 6$ & $1.361(6)$ & $\mathrm{C} 15-\mathrm{C} 16$ & $1.361(8)$ \\
\hline $\mathrm{C} 1-\mathrm{C} 2$ & $1.362(6)$ & $\mathrm{C} 16-\mathrm{C} 17$ & $1.334(9)$ \\
\hline $\mathrm{C} 2-\mathrm{C} 3$ & $1.400(7)$ & $\mathrm{C} 17-\mathrm{C} 18$ & $1.368(8)$ \\
\hline $\mathrm{C} 3-\mathrm{C} 4$ & $1.354(7)$ & $\mathrm{C} 22-\mathrm{C} 23$ & $1.394(6)$ \\
\hline $\mathrm{C} 4-\mathrm{C} 5$ & $1.336(7)$ & $\mathrm{C} 23-\mathrm{C} 24$ & $1.356(6)$ \\
\hline $\mathrm{C} 1-\mathrm{Sn} 1-\mathrm{C} 7$ & $115.20(18)$ & $\mathrm{C} 10-\mathrm{C} 9-\mathrm{C} 8$ & $120.0(6)$ \\
\hline $\mathrm{C} 1-\mathrm{Sn} 1-\mathrm{C} 13$ & $106.68(19)$ & $\mathrm{C} 11-\mathrm{C} 10-\mathrm{C} 9$ & $120.2(5)$ \\
\hline $\mathrm{C} 7-\mathrm{Sn} 1-\mathrm{C} 13$ & $107.59(17)$ & $\mathrm{C} 10-\mathrm{C} 11-\mathrm{C} 12$ & $120.3(5)$ \\
\hline $\mathrm{C} 1-\mathrm{Sn} 1-\mathrm{S} 20$ & $117.09(11)$ & $\mathrm{C} 7-\mathrm{C} 12-\mathrm{C} 11$ & $120.5(5)$ \\
\hline $\mathrm{C} 7-\mathrm{Sn} 1-\mathrm{S} 20$ & $109.65(15)$ & $\mathrm{C} 18-\mathrm{C} 13-\mathrm{C} 14$ & $116.8(5)$ \\
\hline $\mathrm{C} 13-\mathrm{Sn} 1-\mathrm{S} 20$ & $98.87(15)$ & $\mathrm{C} 18-\mathrm{C} 13-\mathrm{Sn} 1$ & $122.7(4)$ \\
\hline $\mathrm{C} 20-\mathrm{S} 20-\mathrm{Sn} 1$ & $94.27(19)$ & $\mathrm{C} 14-\mathrm{C} 13-\mathrm{Sn} 1$ & $120.4(4)$ \\
\hline $\mathrm{C} 20-\mathrm{N} 19-\mathrm{C} 24$ & $114.2(4)$ & $\mathrm{C} 15-\mathrm{C} 14-\mathrm{C} 13$ & $121.3(6)$ \\
\hline $\mathrm{C} 20-\mathrm{N} 21-\mathrm{C} 22$ & $115.6(4)$ & $\mathrm{C} 16-\mathrm{C} 15-\mathrm{C} 14$ & $119.6(7)$ \\
\hline $\mathrm{C} 6-\mathrm{C} 1-\mathrm{C} 2$ & $117.4(5)$ & $\mathrm{C} 17-\mathrm{C} 16-\mathrm{C} 15$ & $120.2(8)$ \\
\hline $\mathrm{C} 6-\mathrm{C} 1-\mathrm{Sn} 1$ & $118.9(5)$ & $\mathrm{C} 16-\mathrm{C} 17-\mathrm{C} 18$ & $120.3(8)$ \\
\hline $\mathrm{C} 2-\mathrm{C} 1-\mathrm{Sn} 1$ & $123.6(4)$ & $\mathrm{C} 17-\mathrm{C} 18-\mathrm{C} 13$ & $121.8(6)$ \\
\hline $\mathrm{C} 1-\mathrm{C} 2-\mathrm{C} 3$ & $121.0(5)$ & $\mathrm{N} 21-\mathrm{C} 20-\mathrm{N} 19$ & $127.9(5)$ \\
\hline $\mathrm{C} 4-\mathrm{C} 3-\mathrm{C} 2$ & $119.3(6)$ & $\mathrm{N} 21-\mathrm{C} 20-\mathrm{S} 20$ & $115.5(4)$ \\
\hline $\mathrm{C} 5-\mathrm{C} 4-\mathrm{C} 3$ & $120.5(6)$ & $\mathrm{N} 19-\mathrm{C} 20-\mathrm{S} 20$ & $116.6(4)$ \\
\hline $\mathrm{C} 4-\mathrm{C} 5-\mathrm{C} 6$ & $119.7(6)$ & $\mathrm{N} 21-\mathrm{C} 22-\mathrm{N} 22$ & $116.4(5)$ \\
\hline $\mathrm{C} 1-\mathrm{C} 6-\mathrm{C} 5$ & $122.0(6)$ & $\mathrm{N} 21-\mathrm{C} 22-\mathrm{C} 23$ & $121.7(5)$ \\
\hline $\mathrm{C} 8-\mathrm{C} 7-\mathrm{C} 12$ & $118.2(5)$ & $\mathrm{N} 22-\mathrm{C} 22-\mathrm{C} 23$ & $121.8(5)$ \\
\hline $\mathrm{C} 8-\mathrm{C} 7-\mathrm{Sn} 1$ & $120.0(4)$ & $\mathrm{C} 24-\mathrm{C} 23-\mathrm{C} 22$ & $116.3(5)$ \\
\hline $\mathrm{C} 12-\mathrm{C} 7-\mathrm{Sn} 1$ & $121.7(4)$ & $\mathrm{N} 19-\mathrm{C} 24-\mathrm{C} 23$ & $124.1(5)$ \\
\hline $\mathrm{C} 7-\mathrm{C} 8-\mathrm{C} 9$ & $120.7(5)$ & & \\
\hline
\end{tabular}

\section{Ph 3 Sn_tHPMT_Cl (2)}

$\begin{array}{llll}\mathrm{Sn} 1-\mathrm{S} 20 & 2.8330(7) & \mathrm{C} 13-\mathrm{C} 14 & 1.409(4) \\ \mathrm{Sn} 1-\mathrm{C} 11 & 2.5192(7) & \mathrm{C} 23-\mathrm{C} 22 & 1.511(4) \\ \mathrm{Sn} 1-\mathrm{C} 1 & 2.136(3) & \mathrm{C} 23-\mathrm{C} 24 & 1.517(4) \\ \mathrm{Sn} 1-\mathrm{C} 7 & 2.140(3) & \mathrm{C} 11-\mathrm{C} 12 & 1.392(4) \\ \mathrm{S} n 1-\mathrm{C} 13 & 2.150(3) & \mathrm{C} 11-\mathrm{C} 10 & 1.390(5) \\ \mathrm{S} 20-\mathrm{C} 20 & 1.738(3) & \mathrm{C} 2-\mathrm{C} 3 & 1.404(5) \\ \mathrm{N} 21-\mathrm{C} 22 & 1.459(4) & \mathrm{C} 18-\mathrm{C} 17 & 1.400(5) \\ \mathrm{N} 21-\mathrm{C} 20 & 1.324(4) & \mathrm{C} 8-\mathrm{C} 9 & 1.383(5)\end{array}$




\begin{tabular}{|c|c|c|c|}
\hline $\mathrm{N} 19-\mathrm{C} 20$ & $1.323(4)$ & $\mathrm{C} 10-\mathrm{C} 9$ & $1.396(5)$ \\
\hline $\mathrm{N} 19-\mathrm{C} 24$ & $1.470(4)$ & $\mathrm{C} 6-\mathrm{C} 5$ & $1.397(4)$ \\
\hline $\mathrm{C} 1-\mathrm{C} 2$ & $1.390(4)$ & $\mathrm{C} 17-\mathrm{C} 16$ & $1.379(5)$ \\
\hline $\mathrm{C} 1-\mathrm{C} 6$ & $1.398(4)$ & $\mathrm{C} 5-\mathrm{C} 4$ & $1.390(6)$ \\
\hline $\mathrm{C} 7-\mathrm{C} 12$ & $1.389(4)$ & $\mathrm{C} 14-\mathrm{C} 15$ & $1.394(5)$ \\
\hline $\mathrm{C} 7-\mathrm{C} 8$ & $1.399(4)$ & $\mathrm{C} 4-\mathrm{C} 3$ & $1.374(6)$ \\
\hline $\mathrm{C} 13-\mathrm{C} 18$ & $1.398(4)$ & $\mathrm{C} 16-\mathrm{C} 15$ & $1.386(6)$ \\
\hline $\mathrm{C} 11-\mathrm{S} n 1-\mathrm{S} 20$ & $171.41(2)$ & $\mathrm{C} 22-\mathrm{C} 23-\mathrm{C} 24$ & $108.7(3)$ \\
\hline $\mathrm{C} 1-\mathrm{Sn} 1-\mathrm{S} 20$ & $85.94(8)$ & $\mathrm{C} 10-\mathrm{C} 11-\mathrm{C} 12$ & $119.9(3)$ \\
\hline $\mathrm{C} 1-\mathrm{Sn} 1-\mathrm{Cl} 1$ & $89.77(8)$ & $\mathrm{C} 1-\mathrm{C} 2-\mathrm{C} 3$ & $120.4(3)$ \\
\hline $\mathrm{C} 1-\mathrm{Sn} 1-\mathrm{C} 7$ & $125.78(11)$ & $\mathrm{C} 7-\mathrm{C} 12-\mathrm{C} 11$ & $121.3(3)$ \\
\hline $\mathrm{C} 1-\mathrm{Sn} 1-\mathrm{C} 13$ & $122.78(11)$ & $\mathrm{C} 13-\mathrm{C} 18-\mathrm{C} 17$ & $121.1(3)$ \\
\hline $\mathrm{C} 7-\mathrm{Sn} 1-\mathrm{S} 20$ & $83.98(8)$ & $\mathrm{N} 21-\mathrm{C} 22-\mathrm{C} 23$ & $108.8(2)$ \\
\hline $\mathrm{C} 7-\mathrm{Sn} 1-\mathrm{C} 11$ & $92.53(8)$ & $\mathrm{C} 9-\mathrm{C} 8-\mathrm{C} 7$ & $120.9(3)$ \\
\hline $\mathrm{C} 7-\mathrm{Sn} 1-\mathrm{C} 13$ & $110.87(11)$ & $\mathrm{C} 11-\mathrm{C} 10-\mathrm{C} 9$ & $119.3(3)$ \\
\hline $\mathrm{C} 13-\mathrm{Sn} 1-\mathrm{S} 20$ & $93.05(8)$ & $\mathrm{N} 21-\mathrm{C} 20-\mathrm{S} 20$ & $119.7(2)$ \\
\hline $\mathrm{C} 13-\mathrm{Sn} 1-\mathrm{Cl1}$ & $95.53(8)$ & $\mathrm{N} 19-\mathrm{C} 20-\mathrm{S} 20$ & $120.1(2)$ \\
\hline $\mathrm{C} 20-\mathrm{S} 20-\mathrm{Sn} 1$ & $100.71(9)$ & $\mathrm{N} 19-\mathrm{C} 20-\mathrm{N} 21$ & $120.2(3)$ \\
\hline $\mathrm{C} 20-\mathrm{N} 21-\mathrm{C} 22$ & $123.0(2)$ & $\mathrm{C} 5-\mathrm{C} 6-\mathrm{C} 1$ & $120.2(3)$ \\
\hline $\mathrm{C} 20-\mathrm{N} 19-\mathrm{C} 24$ & $123.2(3)$ & $\mathrm{C} 16-\mathrm{C} 17-\mathrm{C} 18$ & $120.0(3)$ \\
\hline $\mathrm{C} 2-\mathrm{C} 1-\mathrm{Sn} 1$ & $119.7(2)$ & $\mathrm{C} 4-\mathrm{C} 5-\mathrm{C} 6$ & $120.0(3)$ \\
\hline $\mathrm{C} 2-\mathrm{C} 1-\mathrm{C} 6$ & $119.2(3)$ & $\mathrm{C} 8-\mathrm{C} 9-\mathrm{C} 10$ & $120.3(3)$ \\
\hline $\mathrm{C} 6-\mathrm{C} 1-\mathrm{Sn} 1$ & $121.2(2)$ & $\mathrm{C} 15-\mathrm{C} 14-\mathrm{C} 13$ & $120.4(3)$ \\
\hline $\mathrm{C} 12-\mathrm{C} 7-\mathrm{Sn} 1$ & $122.6(2)$ & $\mathrm{N} 19-\mathrm{C} 24-\mathrm{C} 23$ & $109.7(3)$ \\
\hline $\mathrm{C} 12-\mathrm{C} 7-\mathrm{C} 8$ & $118.3(3)$ & $\mathrm{C} 3-\mathrm{C} 4-\mathrm{C} 5$ & $120.3(3)$ \\
\hline $\mathrm{C} 8-\mathrm{C} 7-\mathrm{Sn} 1$ & $118.9(2)$ & $\mathrm{C} 17-\mathrm{C} 16-\mathrm{C} 15$ & $120.0(3)$ \\
\hline $\mathrm{C} 18-\mathrm{C} 13-\mathrm{Sn} 1$ & $121.5(2)$ & $\mathrm{C} 16-\mathrm{C} 15-\mathrm{C} 14$ & $120.5(3)$ \\
\hline $\mathrm{C} 18-\mathrm{C} 13-\mathrm{C} 14$ & $118.0(3)$ & $\mathrm{C} 4-\mathrm{C} 3-\mathrm{C} 2$ & $120.0(3)$ \\
\hline $\mathrm{C} 14-\mathrm{C} 13-\mathrm{Sn} 1$ & $120.2(2)$ & & \\
\hline
\end{tabular}

\section{Ph 3 Sn_MIMT_Cl (3a)}

$\begin{array}{llll}\mathrm{Sn} 1-\mathrm{C} 11 & 2.4345(8) & \mathrm{C} 7-\mathrm{C} 8 & 1.381(4) \\ \mathrm{S} n 1-\mathrm{C} 1 & 2.142(3) & \mathrm{C} 12-\mathrm{C} 11 & 1.392(5) \\ \mathrm{Sn} 1-\mathrm{C} 13 & 2.111(3) & \mathrm{C} 2-\mathrm{C} 3 & 1.381(5) \\ \mathrm{Sn} 1-\mathrm{C} 7 & 2.138(3) & \mathrm{C} 6-\mathrm{C} 5 & 1.376(5) \\ \mathrm{S} 20-\mathrm{C} 20 & 1.700(3) & \mathrm{C} 22-\mathrm{C} 23 & 1.336(5) \\ \mathrm{N} 21-\mathrm{C} 20 & 1.343(3) & \mathrm{C} 3-\mathrm{C} 4 & 1.367(5) \\ \mathrm{N} 21-\mathrm{C} 22 & 1.376(4) & \mathrm{C} 18-\mathrm{C} 17 & 1.389(6) \\ \mathrm{N} 21-\mathrm{C} 21 & 1.460(4) & \mathrm{C} 8-\mathrm{C} 9 & 1.378(5) \\ \mathrm{N} 19-\mathrm{C} 20 & 1.349(3) & \mathrm{C} 4-\mathrm{C} 5 & 1.365(5)\end{array}$




\begin{tabular}{|c|c|c|c|}
\hline $\mathrm{N} 19-\mathrm{C} 23$ & $1.375(4)$ & $\mathrm{C} 9-\mathrm{C} 10$ & $1.371(6)$ \\
\hline $\mathrm{C} 1-\mathrm{C} 2$ & $1.386(4)$ & $\mathrm{C} 14-\mathrm{C} 15$ & $1.383(6)$ \\
\hline $\mathrm{C} 1-\mathrm{C} 6$ & $1.376(4)$ & $\mathrm{C} 11-\mathrm{C} 10$ & $1.354(6)$ \\
\hline $\mathrm{C} 13-\mathrm{C} 18$ & $1.389(4)$ & $\mathrm{C} 17-\mathrm{C} 16$ & $1.358(8)$ \\
\hline $\mathrm{C} 13-\mathrm{C} 14$ & $1.370(4)$ & $\mathrm{C} 16-\mathrm{C} 15$ & $1.348(8)$ \\
\hline $\mathrm{C} 7-\mathrm{C} 12$ & $1.377(4)$ & & \\
\hline $\mathrm{C} 1-\mathrm{Sn} 1-\mathrm{Cl1}$ & $99.47(8)$ & $\mathrm{N} 21-\mathrm{C} 20-\mathrm{N} 19$ & $105.4(2)$ \\
\hline $\mathrm{C} 13-\mathrm{Sn} 1-\mathrm{C} 11$ & $96.25(8)$ & $\mathrm{N} 19-\mathrm{C} 20-\mathrm{S} 20$ & $127.3(2)$ \\
\hline $\mathrm{C} 13-\mathrm{Sn} 1-\mathrm{C} 1$ & $116.25(11)$ & $\mathrm{C} 7-\mathrm{C} 12-\mathrm{C} 11$ & $120.1(4)$ \\
\hline $\mathrm{C} 13-\mathrm{Sn} 1-\mathrm{C} 7$ & $124.37(11)$ & $\mathrm{C} 3-\mathrm{C} 2-\mathrm{C} 1$ & $120.9(3)$ \\
\hline $\mathrm{C} 7-\mathrm{Sn} 1-\mathrm{Cl} 1$ & $94.72(8)$ & $\mathrm{C} 1-\mathrm{C} 6-\mathrm{C} 5$ & $121.3(3)$ \\
\hline $\mathrm{C} 7-\mathrm{Sn} 1-\mathrm{C} 1$ & $115.30(11)$ & $\mathrm{C} 23-\mathrm{C} 22-\mathrm{N} 21$ & $107.2(3)$ \\
\hline $\mathrm{C} 20-\mathrm{N} 21-\mathrm{C} 22$ & $110.3(3)$ & $\mathrm{C} 4-\mathrm{C} 3-\mathrm{C} 2$ & $120.5(3)$ \\
\hline $\mathrm{C} 20-\mathrm{N} 21-\mathrm{C} 21$ & $125.2(2)$ & $\mathrm{C} 13-\mathrm{C} 18-\mathrm{C} 17$ & $119.4(4)$ \\
\hline $\mathrm{C} 22-\mathrm{N} 21-\mathrm{C} 21$ & $124.5(3)$ & $\mathrm{C} 22-\mathrm{C} 23-\mathrm{N} 19$ & $106.9(3)$ \\
\hline $\mathrm{C} 20-\mathrm{N} 19-\mathrm{C} 23$ & $110.3(3)$ & $\mathrm{C} 9-\mathrm{C} 8-\mathrm{C} 7$ & $121.1(3)$ \\
\hline $\mathrm{C} 2-\mathrm{C} 1-\mathrm{Sn} 1$ & $119.2(2)$ & $\mathrm{C} 5-\mathrm{C} 4-\mathrm{C} 3$ & $119.1(3)$ \\
\hline $\mathrm{C} 6-\mathrm{C} 1-\mathrm{Sn} 1$ & $123.3(2)$ & $\mathrm{C} 10-\mathrm{C} 9-\mathrm{C} 8$ & $119.8(4)$ \\
\hline $\mathrm{C} 6-\mathrm{C} 1-\mathrm{C} 2$ & $117.5(3)$ & $\mathrm{C} 4-\mathrm{C} 5-\mathrm{C} 6$ & $120.7(3)$ \\
\hline $\mathrm{C} 18-\mathrm{C} 13-\mathrm{Sn} 1$ & $120.1(2)$ & $\mathrm{C} 13-\mathrm{C} 14-\mathrm{C} 15$ & $120.2(5)$ \\
\hline $\mathrm{C} 14-\mathrm{C} 13-\mathrm{Sn} 1$ & $120.8(3)$ & $\mathrm{C} 10-\mathrm{C} 11-\mathrm{C} 12$ & $120.5(4)$ \\
\hline $\mathrm{C} 14-\mathrm{C} 13-\mathrm{C} 18$ & $118.9(3)$ & $\mathrm{C} 11-\mathrm{C} 10-\mathrm{C} 9$ & $120.1(4)$ \\
\hline $\mathrm{C} 12-\mathrm{C} 7-\mathrm{Sn} 1$ & $121.3(2)$ & $\mathrm{C} 16-\mathrm{C} 17-\mathrm{C} 18$ & $120.6(5)$ \\
\hline $\mathrm{C} 12-\mathrm{C} 7-\mathrm{C} 8$ & $118.4(3)$ & $\mathrm{C} 15-\mathrm{C} 16-\mathrm{C} 17$ & $120.0(5)$ \\
\hline $\mathrm{C} 8-\mathrm{C} 7-\mathrm{Sn} 1$ & $120.2(2)$ & $\mathrm{C} 16-\mathrm{C} 15-\mathrm{C} 14$ & $120.8(5)$ \\
\hline $\mathrm{N} 21-\mathrm{C} 20-\mathrm{S} 20$ & $127.3(2)$ & & \\
\hline
\end{tabular}

\section{Ph ${ }_{3}$ Sn_MIMT_Cl (3a)}

$\begin{array}{llll}\mathrm{Sn} 1-\mathrm{C} 13 & 2.124(2) & \mathrm{C} 14-\mathrm{C} 15 & 1.379(4) \\ \mathrm{Sn} 1-\mathrm{C} 1 & 2.130(2) & \mathrm{C} 15-\mathrm{C} 16 & 1.385(4) \\ \mathrm{Sn} 1-\mathrm{C} 7 & 2.131(2) & \mathrm{C} 16-\mathrm{C} 17 & 1.375(3) \\ \mathrm{Sn} 1-\mathrm{C} 11 & 2.5801(7) & \mathrm{C} 17-\mathrm{C} 18 & 1.380(3) \\ \mathrm{Sn} 1-\mathrm{S} 20 & 2.7611(6) & \mathrm{S} 20-\mathrm{C} 20 \mathrm{~A} & 1.706(4) \\ \mathrm{C} 1-\mathrm{C} 6 & 1.388(3) & \mathrm{S} 20-\mathrm{C} 20 \mathrm{~B} & 1.762(14) \\ \mathrm{C} 1-\mathrm{C} 2 & 1.401(3) & \mathrm{N} 19 \mathrm{~A}-\mathrm{C} 20 \mathrm{~A} & 1.346(6) \\ \mathrm{C} 2-\mathrm{C} 3 & 1.387(4) & \mathrm{N} 19 \mathrm{~A}-\mathrm{C} 23 \mathrm{~A} & 1.375(5) \\ \mathrm{C} 3-\mathrm{C} 4 & 1.378(4) & \mathrm{C} 20 \mathrm{~A}-\mathrm{N} 21 \mathrm{~A} & 1.350(5) \\ \mathrm{C} 4-\mathrm{C} 5 & 1.377(4) & \mathrm{N} 21 \mathrm{~A}-\mathrm{C} 22 \mathrm{~A} & 1.398(7) \\ \mathrm{C} 5-\mathrm{C} 6 & 1.393(3) & \mathrm{N} 21 \mathrm{~A}-\mathrm{C} 21 \mathrm{~A} & 1.447(9) \\ \mathrm{C} 7-\mathrm{C} 8 & 1.385(3) & \mathrm{C} 22 \mathrm{~A}-\mathrm{C} 23 \mathrm{~A} & 1.312(8) \\ \mathrm{C} 7-\mathrm{C} 12 & 1.391(4) & \mathrm{N} 19 \mathrm{~B}-\mathrm{C} 20 \mathrm{~B} & 1.362(17) \\ \mathrm{C} 8-\mathrm{C} 9 & 1.390(4) & \mathrm{N} 19 \mathrm{~B}-\mathrm{C} 23 \mathrm{~B} & 1.40(2) \\ \mathrm{C} 9-\mathrm{C} 10 & 1.380(5) & \mathrm{C} 20 \mathrm{~B}-\mathrm{N} 21 \mathrm{~B} & 1.308(15)\end{array}$




$$
\begin{aligned}
& \mathrm{C} 10-\mathrm{C} 11 \\
& \mathrm{C} 12-\mathrm{C} 11 \\
& \mathrm{C} 13-\mathrm{C} 18 \\
& \text { C13-C14 } \\
& \text { C13-Sn1-C1 } \\
& \text { C13-Sn1-C7 } \\
& \text { C1-Sn1-C7 } \\
& \text { C13-Sn1-C11 } \\
& \text { C1-Sn1-C11 } \\
& \text { C7-Sn1-C11 } \\
& \text { C13-Sn1-S20 } \\
& \text { C1-Sn1-S20 } \\
& \text { C7-Sn1-S20 } \\
& \text { C11-Sn1-S20 } \\
& \text { C6-C1-C2 } \\
& \text { C6-C1-Sn1 } \\
& \text { C2-C1-Sn1 } \\
& \text { C3-C2-C1 } \\
& \text { C4-C3-C2 } \\
& \text { C3-C4-C5 } \\
& \text { C4-C5-C6 } \\
& \text { C1-C6-C5 } \\
& \text { C8-C7-C12 } \\
& \text { C8-C7-Sn1 } \\
& \text { C12-C7-Sn1 } \\
& \text { C7-C } 8-\mathrm{C} 9 \\
& \text { C10-C9-C8 } \\
& \text { C11-C10-C9 } \\
& \text { C7-C12-C11 } \\
& \text { C10-C11-C12 } \\
& \text { C18-C13-C14 }
\end{aligned}
$$

1.368 (4)

$1.392(3)$

1.383 (3)

1.399 (3)

$116.53(9)$

119.15 (9) 123.93 (9)

93.58 (6)

$91.30(7)$

91.47 (7)

89.53 (6)

$91.58(7)$

82.85 (7)

174.31 (2)

119.1 (2)

$119.56(18)$

$121.34(18)$

120.0 (2)

120.3 (3)

120.2 (2)

120.1 (3)

120.3 (2)

118.7 (2)

120.4 (2)

120.90 (19)

120.7 (3)

119.7 (3)

$120.6(3)$

$120.6(3)$

119.8 (3)

118.4 (2)

N21B-C22B
N21B-C21B
C22B-C23B
C18-C13-Sn1
C14-C13-Sn1
C15-C14-C13
C14-C15-C16
C17-C16-C15
C16-C17-C18
C17-C18-C13
C20A-S20-Sn1
C20B-S20-Sn1
C20A-N19A-C23A
N19A-C20A-N21A
N19A-C20A-S20
N21A-C20A-S20
C20A-N21A-C22A
C20A-N21A-C21A
C22A-N21A-C21A
C23A-C22A-N21A
C22A-C23A-N19A
C20B-N19B-C23B
N21B-C20B-N19B
N21B-C20B-S20
N19B-C20B-S20
C20B-N21B-C22B
C20B-N21B-C21B
C22B-N21B-C21B
C23B-C22B-N21B
C22B-C23B-N19B

1.34 (2)

1.47 (2)

1.346 (19)

$121.32(16)$

120.08 (17)

120.4 (2)

120.4 (2)

119.3 (2)

120.6 (2)

120.9 (2)

105.42 (11)

101.4 (4)

$110.6(5)$

106.1 (4)

$126.3(3)$

127.7 (4)

$108.0(6)$

$125.3(5)$

$126.0(5)$

108.7 (4)

$106.6(4)$

110 (2)

105.5 (19)

$127.6(14)$

126.8 (14)

110.9 (17)

125.5 (17)

123.5 (13)

110.1 (14)

$103.1(16)$

$\mathrm{Ph}_{3} \mathrm{Sn}_{-}(\mathrm{MIMT})_{2}-\mathrm{Ph}_{3} \mathrm{SnCl}_{2}$ (4)

$\begin{array}{llll}\mathrm{C} 25-\mathrm{C} 30 & 1.368(7) & \mathrm{C} 17-\mathrm{C} 16 & 1.364(8) \\ \mathrm{C} 25-\mathrm{C} 26 & 1.362(8) & \mathrm{C} 4-\mathrm{C} 5 & 1.369(9) \\ \mathrm{C} 25-\mathrm{S} 22 & 2.134(4) & \mathrm{C} 15-\mathrm{C} 14 & 1.388(8) \\ \mathrm{C} 30-\mathrm{C} 29 & 1.386(8) & \mathrm{C} 15-\mathrm{C} 16 & 1.361(9) \\ \mathrm{C} 26-\mathrm{C} 27 & 1.403(9) & \mathrm{S} 2-\mathrm{S} 43 & 2.7202(12) \\ \mathrm{C} 36-\mathrm{C} 31 & 1.371(8) & \mathrm{S} 2-\mathrm{C} 19 & 2.148(4) \\ \mathrm{C} 36-\mathrm{C} 35 & 1.408(10) & \mathrm{S} 2-\mathrm{C} 31 & 2.143(5) \\ \mathrm{C} 39-\mathrm{N} 39 & 1.460(7) & \mathrm{S} 2-\mathrm{S} 38 & 2.7394(13) \\ \mathrm{C} 44-\mathrm{N} 44 & 1.463(8) & \mathrm{S} 43-\mathrm{C} 43 & 1.708(5) \\ \mathrm{C} 29-\mathrm{C} 28 & 1.341(11) & \mathrm{C} 19-\mathrm{C} 24 & 1.380(7) \\ \mathrm{C} 28-\mathrm{C} 27 & 1.360(11) & \mathrm{C} 19-\mathrm{C} 20 & 1.388(7) \\ \mathrm{Sn} 1-\mathrm{C} 12 & 2.6257(12) & \mathrm{C} 24-\mathrm{C} 23 & 1.371(8) \\ \mathrm{Sn} 1-\mathrm{C} 11 & 2.6127(12) & \mathrm{C} 20-\mathrm{C} 21 & 1.388(8) \\ \mathrm{Sn} 1-\mathrm{C} 7 & 2.159(4) & \mathrm{C} 43-\mathrm{N} 44 & 1.345(6)\end{array}$




\begin{tabular}{|c|c|c|c|}
\hline $\mathrm{Sn} 1-\mathrm{C} 1$ & $2.139(4)$ & $\mathrm{C} 43-\mathrm{N} 42$ & $1.346(7)$ \\
\hline $\mathrm{Sn} 1-\mathrm{C} 13$ & $2.129(4)$ & $\mathrm{N} 44-\mathrm{C} 45$ & $1.383(8)$ \\
\hline $\mathrm{C} 7-\mathrm{C} 12$ & $1.392(7)$ & $\mathrm{C} 21-\mathrm{C} 22$ & $1.379(9)$ \\
\hline $\mathrm{C} 7-\mathrm{C} 8$ & $1.378(7)$ & $\mathrm{N} 42-\mathrm{C} 46$ & $1.371(7)$ \\
\hline $\mathrm{C} 1-\mathrm{C} 2$ & $1.372(6)$ & $\mathrm{C} 32-\mathrm{C} 33$ & $1.395(10)$ \\
\hline $\mathrm{C} 1-\mathrm{C} 6$ & $1.367(7)$ & $\mathrm{C} 32-\mathrm{C} 31$ & $1.380(8)$ \\
\hline $\mathrm{C} 12-\mathrm{C} 11$ & $1.389(8)$ & $\mathrm{C} 23-\mathrm{C} 22$ & $1.393(9)$ \\
\hline $\mathrm{C} 13-\mathrm{C} 18$ & $1.375(7)$ & $\mathrm{C} 33-\mathrm{C} 34$ & $1.356(12)$ \\
\hline $\mathrm{C} 13-\mathrm{C} 14$ & $1.372(6)$ & $\mathrm{C} 45-\mathrm{C} 46$ & $1.333(9)$ \\
\hline $\mathrm{C} 11-\mathrm{C} 10$ & $1.366(10)$ & $\mathrm{C} 34-\mathrm{C} 35$ & $1.349(12)$ \\
\hline $\mathrm{C} 8-\mathrm{C} 9$ & $1.392(7)$ & $\mathrm{S} 38-\mathrm{C} 38$ & $1.719(6)$ \\
\hline $\mathrm{C} 10-\mathrm{C} 9$ & $1.382(10)$ & $\mathrm{C} 40-\mathrm{N} 39$ & $1.380(8)$ \\
\hline $\mathrm{C} 2-\mathrm{C} 3$ & $1.389(7)$ & $\mathrm{C} 40-\mathrm{C} 41$ & $1.335(9)$ \\
\hline $\mathrm{C} 18-\mathrm{C} 17$ & $1.384(8)$ & N37-C38 & $1.341(7)$ \\
\hline $\mathrm{C} 6-\mathrm{C} 5$ & $1.390(7)$ & N37-C41 & $1.361(8)$ \\
\hline $\mathrm{C} 3-\mathrm{C} 4$ & $1.352(9)$ & $\mathrm{C} 38-\mathrm{N} 39$ & $1.342(6)$ \\
\hline $\mathrm{C} 30-\mathrm{C} 25-\mathrm{Sn} 2$ & $121.9(4)$ & $\mathrm{C} 25-\mathrm{Sn} 2-\mathrm{C} 19$ & $115.86(19)$ \\
\hline $\mathrm{C} 26-\mathrm{C} 25-\mathrm{C} 30$ & $117.7(5)$ & $\mathrm{C} 25-\mathrm{Sn} 2-\mathrm{C} 31$ & $123.0(2)$ \\
\hline $\mathrm{C} 26-\mathrm{C} 25-\mathrm{Sn} 2$ & $120.3(4)$ & $\mathrm{C} 25-\mathrm{Sn} 2-\mathrm{S} 38$ & $93.41(14)$ \\
\hline $\mathrm{C} 25-\mathrm{C} 30-\mathrm{C} 29$ & $121.4(6)$ & $\mathrm{S} 43-\mathrm{Sn} 2-\mathrm{S} 38$ & $169.00(4)$ \\
\hline $\mathrm{C} 25-\mathrm{C} 26-\mathrm{C} 27$ & $121.1(7)$ & $\mathrm{C} 19-\mathrm{Sn} 2-\mathrm{S} 43$ & $95.61(13)$ \\
\hline $\mathrm{C} 31-\mathrm{C} 36-\mathrm{C} 35$ & $120.5(8)$ & $\mathrm{C} 19-\mathrm{Sn} 2-\mathrm{S} 38$ & $95.39(13)$ \\
\hline $\mathrm{C} 28-\mathrm{C} 29-\mathrm{C} 30$ & $120.3(7)$ & $\mathrm{C} 31-\mathrm{Sn} 2-\mathrm{S} 43$ & $91.24(14)$ \\
\hline $\mathrm{C} 29-\mathrm{C} 28-\mathrm{C} 27$ & $120.1(6)$ & $\mathrm{C} 31-\mathrm{Sn} 2-\mathrm{C} 19$ & $121.18(19)$ \\
\hline $\mathrm{C} 28-\mathrm{C} 27-\mathrm{C} 26$ & $119.4(7)$ & $\mathrm{C} 31-\mathrm{Sn} 2-\mathrm{S} 38$ & $82.63(13)$ \\
\hline $\mathrm{C} 11-\mathrm{Sn} 1-\mathrm{Cl} 2$ & $170.10(4)$ & $\mathrm{C} 43-\mathrm{S} 43-\mathrm{Sn} 2$ & $110.05(16)$ \\
\hline $\mathrm{C} 7-\mathrm{Sn} 1-\mathrm{Cl} 2$ & $95.23(13)$ & $\mathrm{C} 24-\mathrm{C} 19-\mathrm{Sn} 2$ & $119.5(3)$ \\
\hline $\mathrm{C} 7-\mathrm{Sn} 1-\mathrm{Cl} 1$ & 94.47 (13) & $\mathrm{C} 24-\mathrm{C} 19-\mathrm{C} 20$ & $119.3(5)$ \\
\hline $\mathrm{C} 1-\mathrm{Sn} 1-\mathrm{Cl} 2$ & $88.48(12)$ & $\mathrm{C} 20-\mathrm{C} 19-\mathrm{Sn} 2$ & $121.2(4)$ \\
\hline $\mathrm{C} 1-\mathrm{Sn} 1-\mathrm{Cl} 1$ & $89.10(12)$ & $\mathrm{C} 23-\mathrm{C} 24-\mathrm{C} 19$ & $120.7(5)$ \\
\hline $\mathrm{C} 1-\mathrm{Sn} 1-\mathrm{C} 7$ & $115.91(17)$ & $\mathrm{C} 21-\mathrm{C} 20-\mathrm{C} 19$ & $120.0(6)$ \\
\hline $\mathrm{C} 13-\mathrm{Sn} 1-\mathrm{Cl} 2$ & $86.35(12)$ & $\mathrm{N} 44-\mathrm{C} 43-\mathrm{S} 43$ & $128.8(4)$ \\
\hline $\mathrm{C} 13-\mathrm{Sn} 1-\mathrm{Cl}$ & $87.77(12)$ & $\mathrm{N} 44-\mathrm{C} 43-\mathrm{N} 42$ & $105.9(4)$ \\
\hline $\mathrm{C} 13-\mathrm{Sn} 1-\mathrm{C} 7$ & $114.79(16)$ & $\mathrm{N} 42-\mathrm{C} 43-\mathrm{S} 43$ & $125.2(4)$ \\
\hline $\mathrm{C} 13-\mathrm{Sn} 1-\mathrm{C} 1$ & $129.29(17)$ & $\mathrm{C} 43-\mathrm{N} 44-\mathrm{C} 44$ & $124.9(5)$ \\
\hline $\mathrm{C} 12-\mathrm{C} 7-\mathrm{Sn} 1$ & $121.1(4)$ & $\mathrm{C} 43-\mathrm{N} 44-\mathrm{C} 45$ & $109.5(5)$ \\
\hline $\mathrm{C} 8-\mathrm{C} 7-\mathrm{Sn} 1$ & $119.6(4)$ & $\mathrm{C} 45-\mathrm{N} 44-\mathrm{C} 44$ & $125.2(5)$ \\
\hline $\mathrm{C} 8-\mathrm{C} 7-\mathrm{C} 12$ & $119.2(5)$ & $\mathrm{C} 22-\mathrm{C} 21-\mathrm{C} 20$ & $120.6(6)$ \\
\hline $\mathrm{C} 2-\mathrm{C} 1-\mathrm{Sn} 1$ & $123.0(3)$ & $\mathrm{C} 43-\mathrm{N} 42-\mathrm{C} 46$ & $110.2(5)$ \\
\hline $\mathrm{C} 6-\mathrm{C} 1-\mathrm{Sn} 1$ & $118.2(3)$ & $\mathrm{C} 31-\mathrm{C} 32-\mathrm{C} 33$ & $120.7(7)$ \\
\hline $\mathrm{C} 6-\mathrm{C} 1-\mathrm{C} 2$ & $118.8(4)$ & $\mathrm{C} 24-\mathrm{C} 23-\mathrm{C} 22$ & $120.5(6)$ \\
\hline
\end{tabular}




$\begin{array}{llll}\mathrm{C} 11-\mathrm{C} 12-\mathrm{C} 7 & 119.9(6) & \mathrm{C} 34-\mathrm{C} 33-\mathrm{C} 32 & 119.3(8) \\ \mathrm{C} 18-\mathrm{C} 13-\mathrm{S} 1 & 124.1(3) & \mathrm{C} 21-\mathrm{C} 22-\mathrm{C} 23 & 118.9(6) \\ \mathrm{C} 14-\mathrm{C} 13-\mathrm{S} 11 & 118.1(4) & \mathrm{C} 36-\mathrm{C} 31-\mathrm{S} 22 & 120.2(5) \\ \mathrm{C} 14-\mathrm{C} 13-\mathrm{C} 18 & 117.8(5) & \mathrm{C} 36-\mathrm{C} 31-\mathrm{C} 32 & 118.6(6) \\ \mathrm{C} 10-\mathrm{C} 11-\mathrm{C} 12 & 120.7(6) & \mathrm{C} 32-\mathrm{C} 31-\mathrm{S} 22 & 121.2(4) \\ \mathrm{C} 7-\mathrm{C} 8-\mathrm{C} 9 & 120.3(6) & \mathrm{C} 46-\mathrm{C} 45-\mathrm{N} 44 & 107.2(5) \\ \mathrm{C} 11-\mathrm{C} 10-\mathrm{C} 9 & 119.8(6) & \mathrm{C} 35-\mathrm{C} 34-\mathrm{C} 33 & 121.6(7) \\ \mathrm{C} 1-\mathrm{C} 2-\mathrm{C} 3 & 119.7(5) & \mathrm{C} 34-\mathrm{C} 35-\mathrm{C} 36 & 119.3(8) \\ \mathrm{C} 13-\mathrm{C} 18-\mathrm{C} 17 & 121.0(5) & \mathrm{C} 45-\mathrm{C} 46-\mathrm{N} 42 & 107.2(6) \\ \mathrm{C} 1-\mathrm{C} 6-\mathrm{C} 5 & 121.5(5) & \mathrm{C} 38-\mathrm{S} 38-\mathrm{S} 2 & 107.14(18) \\ \mathrm{C} 4-\mathrm{C} 3-\mathrm{C} 2 & 120.9(6) & \mathrm{C} 41-\mathrm{C} 40-\mathrm{N} 39 & 107.4(5) \\ \mathrm{C} 16-\mathrm{C} 17-\mathrm{C} 18 & 120.3(6) & \mathrm{C} 38-\mathrm{N} 37-\mathrm{C} 41 & 110.4(5) \\ \mathrm{C} 3-\mathrm{C} 4-\mathrm{C} 5 & 120.2(5) & \mathrm{N} 37-\mathrm{C} 38-\mathrm{S} 38 & 126.4(4) \\ \mathrm{C} 16-\mathrm{C} 15-\mathrm{C} 14 & 120.0(5) & \mathrm{N} 37-\mathrm{C} 38-\mathrm{N} 39 & 106.1(5) \\ \mathrm{C} 4-\mathrm{C} 5-\mathrm{C} 6 & 118.8(6) & \mathrm{N} 39-\mathrm{C} 38-\mathrm{S} 38 & 127.5(4) \\ \mathrm{C} 13-\mathrm{C} 14-\mathrm{C} 15 & 121.2(5) & \mathrm{C} 40-\mathrm{N} 39-\mathrm{C} 39 & 124.4(5) \\ \mathrm{C} 15-\mathrm{C} 16-\mathrm{C} 17 & 119.5(5) & \mathrm{C} 38-\mathrm{N} 39-\mathrm{C} 39 & 126.3(6) \\ \mathrm{C} 10-\mathrm{C} 9-\mathrm{C} 8 & 120.0(6) & \mathrm{C} 38-\mathrm{N} 39-\mathrm{C} 40 & 109.1(5) \\ \mathrm{C} 25-\mathrm{S} 2-\mathrm{S} 43 & 82.23(14) & \mathrm{C} 40-\mathrm{C} 41-\mathrm{N} 37 & 107.0(6) \\ & & & \end{array}$

\section{$\mathrm{SnPh}_{3} \mathrm{Cl}$ _DMF (5)}

$\begin{array}{llll}\mathrm{Sn} 1-\mathrm{C} 12 & 2.4964(5) & \mathrm{C} 10-\mathrm{C} 9 & 1.381(4) \\ \mathrm{Sn} 1-\mathrm{O} 19 & 2.3561(14) & \mathrm{C} 18-\mathrm{C} 13 & 1.391(3) \\ \mathrm{Sn} 1-\mathrm{C} 1 & 2.136(2) & \mathrm{C} 1-\mathrm{C} 6 & 1.385(3) \\ \mathrm{Sn} 1-\mathrm{C} 7 & 2.126(2) & \mathrm{C} 1-\mathrm{C} 2 & 1.394(3) \\ \mathrm{S} 1-\mathrm{C} 13 & 2.131(2) & \mathrm{C} 5-\mathrm{C} 4 & 1.376(4) \\ \mathrm{O} 19-\mathrm{C} 20 & 1.245(3) & \mathrm{C} 5-\mathrm{C} 6 & 1.396(3) \\ \mathrm{N} 21-\mathrm{C} 23 & 1.450(3) & \mathrm{C} 11-\mathrm{C} 12 & 1.385(3) \\ \mathrm{N} 21-\mathrm{C} 22 & 1.451(3) & \mathrm{C} 14-\mathrm{C} 13 & 1.399(3) \\ \mathrm{N} 21-\mathrm{C} 20 & 1.314(3) & \mathrm{C} 7-\mathrm{C} 12 & 1.384(3) \\ \mathrm{C} 16-\mathrm{C} 17 & 1.387(3) & \mathrm{C} 7-\mathrm{C} 8 & 1.400(3) \\ \mathrm{C} 16-\mathrm{C} 15 & 1.383(3) & \mathrm{C} 9-\mathrm{C} 8 & 1.382(3) \\ \mathrm{C} 17-\mathrm{C} 18 & 1.387(3) & \mathrm{C} 4-\mathrm{C} 3 & 1.373(4) \\ \mathrm{C} 15-\mathrm{C} 14 & 1.385(3) & \mathrm{C} 3-\mathrm{C} 2 & 1.390(3) \\ \mathrm{C} 10-\mathrm{C} 11 & 1.382(3) & & \\ & & & 118.0(2) \\ \mathrm{O} 19-\mathrm{Sn} 1-\mathrm{C} 12 & 178.07(4) & \mathrm{C} 6-\mathrm{C} 1-\mathrm{C} 2 & 121.73(18) \\ \mathrm{C} 1-\mathrm{S} 1-\mathrm{C} 12 & 94.57(6) & \mathrm{C} 2-\mathrm{C} 1-\mathrm{Sn} 1 & \\ \mathrm{C} 1-\mathrm{S} 1-\mathrm{O} 19 & 84.56(7) & \mathrm{C} 4-\mathrm{C} 5-\mathrm{C} 6 & \end{array}$




$\begin{array}{llll}\mathrm{C} 7-\mathrm{Sn} 1-\mathrm{C} 12 & 95.64(6) & \mathrm{C} 10-\mathrm{C} 11-\mathrm{C} 12 & 120.0(2) \\ \mathrm{C} 7-\mathrm{S} 1-\mathrm{O} 19 & 86.29(7) & \mathrm{C} 15-\mathrm{C} 14-\mathrm{C} 13 & 121.0(2) \\ \mathrm{C} 7-\mathrm{S} 1-\mathrm{C} 1 & 118.42(8) & \mathrm{C} 12-\mathrm{C} 7-\mathrm{S} 11 & 121.66(16) \\ \mathrm{C} 7-\mathrm{S} 1-\mathrm{C} 13 & 117.80(8) & \mathrm{C} 12-\mathrm{C} 7-\mathrm{C} 8 & 117.9(2) \\ \mathrm{C} 13-\mathrm{S} 1-\mathrm{C} 12 & 93.57(6) & \mathrm{C} 8-\mathrm{C} 7-\mathrm{S} 11 & 120.44(17) \\ \mathrm{C} 13-\mathrm{S} 1-\mathrm{O} 19 & 85.44(7) & \mathrm{C} 7-\mathrm{C} 12-\mathrm{C} 11 & 121.3(2) \\ \mathrm{C} 13-\mathrm{S} 1-\mathrm{C} 1 & 121.88(8) & \mathrm{O} 19-\mathrm{C} 20-\mathrm{N} 21 & 123.9(2) \\ \mathrm{C} 20-\mathrm{O} 19-\mathrm{S} 1 & 124.63(13) & \mathrm{C} 10-\mathrm{C} 9-\mathrm{C} 8 & 120.2(2) \\ \mathrm{C} 23-\mathrm{N} 21-\mathrm{C} 22 & 117.41(18) & \mathrm{C} 3-\mathrm{C} 4-\mathrm{C} 5 & 119.7(2) \\ \mathrm{C} 20-\mathrm{N} 21-\mathrm{C} 23 & 120.1(2) & \mathrm{C} 18-\mathrm{C} 13-\mathrm{S} 1 & 121.71(16) \\ \mathrm{C} 20-\mathrm{N} 21-\mathrm{C} 22 & 122.38(19) & \mathrm{C} 18-\mathrm{C} 13-\mathrm{C} 14 & 118.1(2) \\ \mathrm{C} 15-\mathrm{C} 16-\mathrm{C} 17 & 119.5(2) & \mathrm{C} 14-\mathrm{C} 13-\mathrm{S} 1 & 120.14(16) \\ \mathrm{C} 16-\mathrm{C} 17-\mathrm{C} 18 & 120.3(2) & \mathrm{C} 4-\mathrm{C} 3-\mathrm{C} 2 & 120.4(2) \\ \mathrm{C} 16-\mathrm{C} 15-\mathrm{C} 14 & 120.2(2) & \mathrm{C} 1-\mathrm{C} 6-\mathrm{C} 5 & 121.1(2) \\ \mathrm{C} 9-\mathrm{C} 10-\mathrm{C} 11 & 119.7(2) & \mathrm{C} 9-\mathrm{C} 8-\mathrm{C} 7 & 121.0(2) \\ \mathrm{C} 17-\mathrm{C} 18-\mathrm{C} 13 & 120.9(2) & \mathrm{C} 3-\mathrm{C} 2-\mathrm{C} 1 & 120.7(2) \\ \mathrm{C} 6-\mathrm{C} 1-\mathrm{S} 11 & 120.24(16) & & \end{array}$

\section{$\mathrm{Ph}_{3} \mathrm{Sn} \_D M S O \_C l$ (6a)}

$\begin{array}{llll}\mathrm{Sn} 2-\mathrm{C} 12 & 2.4818(16) & \mathrm{C} 1-\mathrm{C} 6 & 1.385(9) \\ \mathrm{S} n 2-\mathrm{O} 2 & 2.426(4) & \mathrm{C} 11-\mathrm{C} 12 & 1.402(8) \\ \mathrm{S} n 2-\mathrm{C} 21 & 2.125(5) & \mathrm{C} 11-\mathrm{C} 10 & 1.369(10) \\ \mathrm{S} n 2-\mathrm{C} 33 & 2.129(6) & \mathrm{C} 3-\mathrm{C} 2 & 1.391(9) \\ \mathrm{S} 2-\mathrm{C} 27 & 2.123(6) & \mathrm{C} 3-\mathrm{C} 4 & 1.389(10) \\ \mathrm{S} n 1-\mathrm{C} 11 & 2.5207(16) & \mathrm{C} 27-\mathrm{C} 32 & 1.404(8) \\ \mathrm{S} 1-\mathrm{O} 1 & 2.336(4) & \mathrm{C} 27-\mathrm{C} 28 & 1.378(9) \\ \mathrm{S} 1-\mathrm{C} 13 & 2.143(6) & \mathrm{C} 25-\mathrm{C} 24 & 1.390(9) \\ \mathrm{S} 1-\mathrm{C} 7 & 2.139(6) & \mathrm{C} 34-\mathrm{C} 35 & 1.391(8) \\ \mathrm{S} 1-\mathrm{C} 1 & 2.136(6) & \mathrm{C} 22-\mathrm{C} 23 & 1.395(8) \\ \mathrm{S} 1-\mathrm{O} 1 & 1.525(4) & \mathrm{C} 18-\mathrm{C} 17 & 1.371(9) \\ \mathrm{S} 1-\mathrm{C} 20 & 1.791(6) & \mathrm{C} 32-\mathrm{C} 31 & 1.395(9) \\ \mathrm{S} 1-\mathrm{C} 19 & 1.776(6) & \mathrm{C} 35-\mathrm{C} 36 & 1.373(10) \\ \mathrm{S} 2-\mathrm{O} 2 & 1.528(4) & \mathrm{C} 4-\mathrm{C} 5 & 1.382(9) \\ \mathrm{S} 2-\mathrm{C} 40 & 1.774(6) & \mathrm{C} 6-\mathrm{C} 5 & 1.392(9) \\ \mathrm{S} 2-\mathrm{C} 39 & 1.782(6) & \mathrm{C} 15-\mathrm{C} 14 & 1.373(9) \\ \mathrm{C} 21-\mathrm{C} 26 & 1.391(8) & \mathrm{C} 15-\mathrm{C} 16 & 1.391(8) \\ \mathrm{C} 21-\mathrm{C} 22 & 1.390(8) & \mathrm{C} 29-\mathrm{C} 30 & 1.395(9) \\ \mathrm{C} 13-\mathrm{C} 18 & 1.403(8) & \mathrm{C} 29-\mathrm{C} 28 & 1.390(9) \\ \mathrm{C} 13-\mathrm{C} 14 & 1.405(9) & \mathrm{C} 30-\mathrm{C} 31 & 1.385(10) \\ \mathrm{C} 26-\mathrm{C} 25 & 1.396(8) & \mathrm{C} 23-\mathrm{C} 24 & 1.373(9) \\ \mathrm{C} 33-\mathrm{C} 34 & 1.392(8) & \mathrm{C} 38-\mathrm{C} 37 & 1.408(9) \\ & & & \end{array}$




\begin{tabular}{|c|c|c|c|}
\hline $\mathrm{C} 33-\mathrm{C} 38$ & $1.393(9)$ & $\mathrm{C} 17-\mathrm{C} 16$ & $1.391(10)$ \\
\hline $\mathrm{C} 7-\mathrm{C} 12$ & $1.398(8)$ & $\mathrm{C} 8-\mathrm{C} 9$ & $1.402(8)$ \\
\hline $\mathrm{C} 7-\mathrm{C} 8$ & $1.378(9)$ & $\mathrm{C} 9-\mathrm{C} 10$ & $1.389(9)$ \\
\hline $\mathrm{C} 1-\mathrm{C} 2$ & $1.396(8)$ & $\mathrm{C} 36-\mathrm{C} 37$ & $1.396(9)$ \\
\hline $\mathrm{O} 2-\mathrm{Sn} 2-\mathrm{Cl} 2$ & $175.82(12)$ & $\mathrm{C} 12-\mathrm{C} 7-\mathrm{Sn} 1$ & $120.8(5)$ \\
\hline $\mathrm{C} 21-\mathrm{Sn} 2-\mathrm{Cl} 2$ & $92.95(16)$ & $\mathrm{C} 8-\mathrm{C} 7-\mathrm{Sn} 1$ & $120.4(5)$ \\
\hline $\mathrm{C} 21-\mathrm{Sn} 2-\mathrm{O} 2$ & $85.11(17)$ & $\mathrm{C} 8-\mathrm{C} 7-\mathrm{C} 12$ & $118.7(6)$ \\
\hline $\mathrm{C} 21-\mathrm{Sn} 2-\mathrm{C} 33$ & $127.6(2)$ & $\mathrm{C} 2-\mathrm{C} 1-\mathrm{Sn} 1$ & $121.5(5)$ \\
\hline $\mathrm{C} 33-\mathrm{Sn} 2-\mathrm{Cl} 2$ & $94.90(17)$ & $\mathrm{C} 6-\mathrm{C} 1-\mathrm{Sn} 1$ & $120.1(4)$ \\
\hline $\mathrm{C} 33-\mathrm{Sn} 2-\mathrm{O} 2$ & 83.40 (19) & $\mathrm{C} 6-\mathrm{C} 1-\mathrm{C} 2$ & $118.4(6)$ \\
\hline $\mathrm{C} 27-\mathrm{Sn} 2-\mathrm{Cl} 2$ & $97.34(18)$ & $\mathrm{C} 10-\mathrm{C} 11-\mathrm{C} 12$ & $119.9(6)$ \\
\hline $\mathrm{C} 27-\mathrm{Sn} 2-\mathrm{O} 2$ & $86.8(2)$ & $\mathrm{C} 7-\mathrm{C} 12-\mathrm{C} 11$ & $120.2(7)$ \\
\hline $\mathrm{C} 27-\mathrm{Sn} 2-\mathrm{C} 21$ & $119.2(2)$ & $\mathrm{C} 4-\mathrm{C} 3-\mathrm{C} 2$ & $119.7(6)$ \\
\hline $\mathrm{C} 27-\mathrm{Sn} 2-\mathrm{C} 33$ & $111.0(2)$ & $\mathrm{C} 3-\mathrm{C} 2-\mathrm{C} 1$ & $120.9(6)$ \\
\hline $\mathrm{O} 1-\mathrm{Sn} 1-\mathrm{Cl} 1$ & $179.04(10)$ & $\mathrm{C} 32-\mathrm{C} 27-\mathrm{Sn} 2$ & $119.3(5)$ \\
\hline $\mathrm{C} 13-\mathrm{Sn} 1-\mathrm{Cl} 1$ & 93.70 (17) & $\mathrm{C} 28-\mathrm{C} 27-\mathrm{Sn} 2$ & $122.3(4)$ \\
\hline $\mathrm{C} 13-\mathrm{Sn} 1-\mathrm{O} 1$ & $86.4(2)$ & $\mathrm{C} 28-\mathrm{C} 27-\mathrm{C} 32$ & $118.4(6)$ \\
\hline $\mathrm{C} 7-\mathrm{Sn} 1-\mathrm{Cl1}$ & $91.72(15)$ & $\mathrm{C} 24-\mathrm{C} 25-\mathrm{C} 26$ & $119.6(6)$ \\
\hline $\mathrm{C} 7-\mathrm{Sn} 1-\mathrm{O} 1$ & $87.46(18)$ & $\mathrm{C} 35-\mathrm{C} 34-\mathrm{C} 33$ & $120.7(6)$ \\
\hline $\mathrm{C} 7-\mathrm{Sn} 1-\mathrm{C} 13$ & $126.0(2)$ & $\mathrm{C} 21-\mathrm{C} 22-\mathrm{C} 23$ & $120.3(6)$ \\
\hline $\mathrm{C} 1-\mathrm{Sn} 1-\mathrm{Cl} 1$ & $93.81(16)$ & $\mathrm{C} 17-\mathrm{C} 18-\mathrm{C} 13$ & $121.2(7)$ \\
\hline $\mathrm{C} 1-\mathrm{Sn} 1-\mathrm{O} 1$ & $86.96(19)$ & $\mathrm{C} 31-\mathrm{C} 32-\mathrm{C} 27$ & $120.6(6)$ \\
\hline $\mathrm{C} 1-\mathrm{Sn} 1-\mathrm{C} 13$ & $120.3(2)$ & $\mathrm{C} 36-\mathrm{C} 35-\mathrm{C} 34$ & $120.2(6)$ \\
\hline $\mathrm{C} 1-\mathrm{Sn} 1-\mathrm{C} 7$ & $112.8(2)$ & $\mathrm{C} 5-\mathrm{C} 4-\mathrm{C} 3$ & $120.1(6)$ \\
\hline $\mathrm{O} 1-\mathrm{S} 1-\mathrm{C} 20$ & $104.9(3)$ & $\mathrm{C} 1-\mathrm{C} 6-\mathrm{C} 5$ & $121.1(6)$ \\
\hline $\mathrm{O} 1-\mathrm{S} 1-\mathrm{C} 19$ & $104.8(3)$ & $\mathrm{C} 14-\mathrm{C} 15-\mathrm{C} 16$ & $120.5(7)$ \\
\hline $\mathrm{C} 19-\mathrm{S} 1-\mathrm{C} 20$ & $98.0(3)$ & $\mathrm{C} 28-\mathrm{C} 29-\mathrm{C} 30$ & $119.3(7)$ \\
\hline $\mathrm{O} 2-\mathrm{S} 2-\mathrm{C} 40$ & $105.3(3)$ & $\mathrm{C} 31-\mathrm{C} 30-\mathrm{C} 29$ & $120.0(6)$ \\
\hline $\mathrm{O} 2-\mathrm{S} 2-\mathrm{C} 39$ & $105.3(3)$ & $\mathrm{C} 30-\mathrm{C} 31-\mathrm{C} 32$ & $119.9(6)$ \\
\hline $\mathrm{C} 40-\mathrm{S} 2-\mathrm{C} 39$ & $98.6(3)$ & $\mathrm{C} 15-\mathrm{C} 14-\mathrm{C} 13$ & $121.1(6)$ \\
\hline $\mathrm{S} 1-\mathrm{O} 1-\mathrm{Sn} 1$ & $132.6(2)$ & $\mathrm{C} 24-\mathrm{C} 23-\mathrm{C} 22$ & $120.3(6)$ \\
\hline $\mathrm{S} 2-\mathrm{O} 2-\mathrm{Sn} 2$ & $119.5(3)$ & $\mathrm{C} 4-\mathrm{C} 5-\mathrm{C} 6$ & $119.8(6)$ \\
\hline $\mathrm{C} 26-\mathrm{C} 21-\mathrm{Sn} 2$ & $118.1(4)$ & $\mathrm{C} 33-\mathrm{C} 38-\mathrm{C} 37$ & $120.4(6)$ \\
\hline $\mathrm{C} 22-\mathrm{C} 21-\mathrm{Sn} 2$ & $122.8(4)$ & $\mathrm{C} 23-\mathrm{C} 24-\mathrm{C} 25$ & $120.1(5)$ \\
\hline $\mathrm{C} 22-\mathrm{C} 21-\mathrm{C} 26$ & $119.1(5)$ & $\mathrm{C} 27-\mathrm{C} 28-\mathrm{C} 29$ & $121.8(6)$ \\
\hline $\mathrm{C} 18-\mathrm{C} 13-\mathrm{Sn} 1$ & $122.1(5)$ & $\mathrm{C} 18-\mathrm{C} 17-\mathrm{C} 16$ & $120.5(6)$ \\
\hline $\mathrm{C} 18-\mathrm{C} 13-\mathrm{C} 14$ & $117.5(6)$ & $\mathrm{C} 7-\mathrm{C} 8-\mathrm{C} 9$ & $121.6(6)$ \\
\hline $\mathrm{C} 14-\mathrm{C} 13-\mathrm{Sn} 1$ & $120.4(4)$ & $\mathrm{C} 10-\mathrm{C} 9-\mathrm{C} 8$ & $118.5(7)$ \\
\hline $\mathrm{C} 21-\mathrm{C} 26-\mathrm{C} 25$ & $120.5(6)$ & $\mathrm{C} 17-\mathrm{C} 16-\mathrm{C} 15$ & $119.1(7)$ \\
\hline $\mathrm{C} 34-\mathrm{C} 33-\mathrm{Sn} 2$ & $117.9(5)$ & $\mathrm{C} 35-\mathrm{C} 36-\mathrm{C} 37$ & $120.5(6)$ \\
\hline $\mathrm{C} 34-\mathrm{C} 33-\mathrm{C} 38$ & $119.0(6)$ & $\mathrm{C} 11-\mathrm{C} 10-\mathrm{C} 9$ & $121.1(6)$ \\
\hline
\end{tabular}


$\mathrm{C} 38-\mathrm{C} 33-\mathrm{Sn} 2 \quad 123.1(4) \quad \mathrm{C} 36-\mathrm{C} 37-\mathrm{C} 38 \quad 119.1(7)$

\section{$\mathrm{Ph}_{3} \mathrm{Sn} \_D M S O \_C l(6 b)$}

\begin{tabular}{|c|c|c|c|}
\hline $\mathrm{Sn} 2-\mathrm{Cl} 2$ & $2.4936(13)$ & $\mathrm{C} 12-\mathrm{C} 7$ & $1.364(9)$ \\
\hline $\mathrm{Sn} 2-\mathrm{O} 2$ & $2.366(4)$ & $\mathrm{C} 12-\mathrm{C} 11$ & $1.420(9)$ \\
\hline $\mathrm{Sn} 2-\mathrm{C} 33$ & $2.139(6)$ & $\mathrm{C} 17-\mathrm{C} 18$ & $1.387(9)$ \\
\hline $\mathrm{Sn} 2-\mathrm{C} 21$ & $2.126(5)$ & $\mathrm{C} 18-\mathrm{C} 13$ & $1.408(8)$ \\
\hline $\mathrm{Sn} 2-\mathrm{C} 27$ & $2.164(6)$ & $\mathrm{C} 26-\mathrm{C} 21$ & $1.388(9)$ \\
\hline $\mathrm{Sn} 1-\mathrm{Cl1}$ & $2.4903(13)$ & $\mathrm{C} 24-\mathrm{C} 23$ & $1.377(11)$ \\
\hline $\mathrm{Sn} 1-\mathrm{O} 1$ & $2.330(4)$ & $\mathrm{C} 13-\mathrm{C} 14$ & $1.397(8)$ \\
\hline $\mathrm{Sn} 1-\mathrm{C} 1$ & $2.138(7)$ & $\mathrm{C} 21-\mathrm{C} 22$ & $1.395(8)$ \\
\hline $\mathrm{Sn} 1-\mathrm{C} 13$ & $2.134(6)$ & $\mathrm{C} 27-\mathrm{C} 28$ & $1.371(9)$ \\
\hline $\mathrm{Sn} 1-\mathrm{C} 7$ & $2.167(6)$ & $\mathrm{C} 27-\mathrm{C} 32$ & $1.393(9)$ \\
\hline $\mathrm{S} 2-\mathrm{O} 2$ & $1.516(4)$ & $\mathrm{C} 28-\mathrm{C} 29$ & $1.405(10)$ \\
\hline $\mathrm{S} 2-\mathrm{C} 40$ & $1.781(7)$ & $\mathrm{C} 23-\mathrm{C} 22$ & $1.411(10)$ \\
\hline $\mathrm{S} 2-\mathrm{C} 39$ & $1.789(10)$ & $\mathrm{C} 38-\mathrm{C} 37$ & $1.387(10)$ \\
\hline $\mathrm{S} 1-\mathrm{O} 1$ & $1.510(5)$ & $\mathrm{C} 8-\mathrm{C} 7$ & $1.364(9)$ \\
\hline $\mathrm{S} 1-\mathrm{C} 19$ & $1.767(7)$ & $\mathrm{C} 8-\mathrm{C} 9$ & $1.426(9)$ \\
\hline $\mathrm{S} 1-\mathrm{C} 20$ & $1.769(9)$ & $\mathrm{C} 11-\mathrm{C} 10$ & $1.371(10)$ \\
\hline $\mathrm{C} 33-\mathrm{C} 34$ & $1.388(8)$ & $\mathrm{C} 37-\mathrm{C} 36$ & $1.378(12)$ \\
\hline $\mathrm{C} 33-\mathrm{C} 38$ & $1.413(8)$ & $\mathrm{C} 9-\mathrm{C} 10$ & $1.365(11)$ \\
\hline $\mathrm{C} 15-\mathrm{C} 16$ & $1.387(9)$ & $\mathrm{C} 5-\mathrm{C} 6$ & $1.386(10)$ \\
\hline $\mathrm{C} 15-\mathrm{C} 14$ & $1.390(9)$ & $\mathrm{C} 5-\mathrm{C} 4$ & $1.374(12)$ \\
\hline $\mathrm{C} 16-\mathrm{C} 17$ & $1.383(9)$ & $\mathrm{C} 2-\mathrm{C} 3$ & $1.385(11)$ \\
\hline $\mathrm{C} 34-\mathrm{C} 35$ & $1.394(10)$ & $\mathrm{C} 29-\mathrm{C} 30$ & $1.398(12)$ \\
\hline $\mathrm{C} 1-\mathrm{C} 6$ & $1.397(10)$ & $\mathrm{C} 32-\mathrm{C} 31$ & $1.395(10)$ \\
\hline $\mathrm{C} 1-\mathrm{C} 2$ & $1.387(9)$ & $\mathrm{C} 35-\mathrm{C} 36$ & $1.372(11)$ \\
\hline $\mathrm{C} 25-\mathrm{C} 26$ & $1.402(9)$ & $\mathrm{C} 31-\mathrm{C} 30$ & $1.374(12)$ \\
\hline $\mathrm{C} 25-\mathrm{C} 24$ & $1.379(10)$ & $\mathrm{C} 3-\mathrm{C} 4$ & $1.392(13)$ \\
\hline $\mathrm{O} 2-\mathrm{Sn} 2-\mathrm{Cl} 2$ & $178.50(12)$ & $\mathrm{C} 7-\mathrm{C} 12-\mathrm{C} 11$ & $120.5(6)$ \\
\hline $\mathrm{C} 33-\mathrm{Sn} 2-\mathrm{Cl} 2$ & $93.96(15)$ & $\mathrm{C} 16-\mathrm{C} 17-\mathrm{C} 18$ & $120.1(6)$ \\
\hline $\mathrm{C} 33-\mathrm{Sn} 2-\mathrm{O} 2$ & $84.75(18)$ & $\mathrm{C} 17-\mathrm{C} 18-\mathrm{C} 13$ & $121.4(5)$ \\
\hline $\mathrm{C} 33-\mathrm{Sn} 2-\mathrm{C} 27$ & $122.3(2)$ & $\mathrm{C} 21-\mathrm{C} 26-\mathrm{C} 25$ & $121.1(6)$ \\
\hline $\mathrm{C} 21-\mathrm{Sn} 2-\mathrm{C} 12$ & $95.24(15)$ & $\mathrm{C} 23-\mathrm{C} 24-\mathrm{C} 25$ & $121.8(6)$ \\
\hline $\mathrm{C} 21-\mathrm{Sn} 2-\mathrm{O} 2$ & $84.68(18)$ & $\mathrm{C} 18-\mathrm{C} 13-\mathrm{Sn} 1$ & $120.3(4)$ \\
\hline $\mathrm{C} 21-\mathrm{Sn} 2-\mathrm{C} 33$ & $116.9(2)$ & $\mathrm{C} 14-\mathrm{C} 13-\mathrm{Sn} 1$ & $122.4(4)$ \\
\hline $\mathrm{C} 21-\mathrm{Sn} 2-\mathrm{C} 27$ & $119.0(2)$ & $\mathrm{C} 14-\mathrm{C} 13-\mathrm{C} 18$ & $117.3(6)$ \\
\hline $\mathrm{C} 27-\mathrm{Sn} 2-\mathrm{Cl} 2$ & $94.02(16)$ & $\mathrm{C} 26-\mathrm{C} 21-\mathrm{Sn} 2$ & $121.0(4)$ \\
\hline $\mathrm{C} 27-\mathrm{Sn} 2-\mathrm{O} 2$ & $87.33(19)$ & $\mathrm{C} 26-\mathrm{C} 21-\mathrm{C} 22$ & $118.7(5)$ \\
\hline $\mathrm{O} 1-\mathrm{Sn} 1-\mathrm{Cl} 1$ & $178.43(14)$ & $\mathrm{C} 22-\mathrm{C} 21-\mathrm{Sn} 2$ & $120.2(4)$ \\
\hline
\end{tabular}




\begin{tabular}{|c|c|c|c|}
\hline $\mathrm{C} 1-\mathrm{Sn} 1-\mathrm{C} 11$ & $92.10(16)$ & $\mathrm{C} 28-\mathrm{C} 27-\mathrm{Sn} 2$ & $120.4(5)$ \\
\hline $\mathrm{C} 1-\mathrm{Sn} 1-\mathrm{O} 1$ & $88.2(2)$ & $\mathrm{C} 28-\mathrm{C} 27-\mathrm{C} 32$ & $120.3(6)$ \\
\hline $\mathrm{C} 1-\mathrm{Sn} 1-\mathrm{C} 7$ & $117.3(3)$ & $\mathrm{C} 32-\mathrm{C} 27-\mathrm{Sn} 2$ & $119.2(5)$ \\
\hline $\mathrm{C} 13-\mathrm{Sn} 1-\mathrm{Cl} 1$ & $92.63(15)$ & $\mathrm{C} 27-\mathrm{C} 28-\mathrm{C} 29$ & $120.8(7)$ \\
\hline $\mathrm{C} 13-\mathrm{Sn} 1-\mathrm{O} 1$ & 85.89 (19) & $\mathrm{C} 24-\mathrm{C} 23-\mathrm{C} 22$ & $118.8(6)$ \\
\hline $\mathrm{C} 13-\mathrm{Sn} 1-\mathrm{C} 1$ & $122.0(2)$ & $\mathrm{C} 37-\mathrm{C} 38-\mathrm{C} 33$ & $120.5(6)$ \\
\hline $\mathrm{C} 13-\mathrm{Sn} 1-\mathrm{C} 7$ & $119.4(2)$ & $\mathrm{C} 7-\mathrm{C} 8-\mathrm{C} 9$ & $119.6(6)$ \\
\hline $\mathrm{C} 7-\mathrm{Sn} 1-\mathrm{C} 11$ & $96.71(17)$ & $\mathrm{C} 12-\mathrm{C} 7-\mathrm{Sn} 1$ & $118.8(4)$ \\
\hline $\mathrm{C} 7-\mathrm{Sn} 1-\mathrm{O} 1$ & $84.5(2)$ & $\mathrm{C} 12-\mathrm{C} 7-\mathrm{C} 8$ & $120.7(6)$ \\
\hline $\mathrm{O} 2-\mathrm{S} 2-\mathrm{C} 40$ & $103.6(3)$ & $\mathrm{C} 8-\mathrm{C} 7-\mathrm{Sn} 1$ & $120.5(5)$ \\
\hline $\mathrm{O} 2-\mathrm{S} 2-\mathrm{C} 39$ & $105.3(4)$ & $\mathrm{C} 15-\mathrm{C} 14-\mathrm{C} 13$ & $121.2(6)$ \\
\hline $\mathrm{C} 40-\mathrm{S} 2-\mathrm{C} 39$ & $97.4(4)$ & $\mathrm{C} 10-\mathrm{C} 11-\mathrm{C} 12$ & $118.2(7)$ \\
\hline $\mathrm{O} 1-\mathrm{S} 1-\mathrm{C} 19$ & $105.1(3)$ & $\mathrm{C} 36-\mathrm{C} 37-\mathrm{C} 38$ & $120.2(7)$ \\
\hline $\mathrm{O} 1-\mathrm{S} 1-\mathrm{C} 20$ & $105.2(4)$ & $\mathrm{C} 10-\mathrm{C} 9-\mathrm{C} 8$ & $119.0(6)$ \\
\hline $\mathrm{C} 19-\mathrm{S} 1-\mathrm{C} 20$ & $98.3(5)$ & $\mathrm{C} 4-\mathrm{C} 5-\mathrm{C} 6$ & $121.0(8)$ \\
\hline $\mathrm{S} 2-\mathrm{O} 2-\mathrm{Sn} 2$ & $130.0(2)$ & $\mathrm{C} 5-\mathrm{C} 6-\mathrm{C} 1$ & $119.9(7)$ \\
\hline $\mathrm{S} 1-\mathrm{O} 1-\mathrm{Sn} 1$ & $135.7(3)$ & $\mathrm{C} 9-\mathrm{C} 10-\mathrm{C} 11$ & $121.9(7)$ \\
\hline $\mathrm{C} 34-\mathrm{C} 33-\mathrm{Sn} 2$ & $121.4(4)$ & $\mathrm{C} 3-\mathrm{C} 2-\mathrm{C} 1$ & $120.4(7)$ \\
\hline $\mathrm{C} 34-\mathrm{C} 33-\mathrm{C} 38$ & $118.0(6)$ & $\mathrm{C} 30-\mathrm{C} 29-\mathrm{C} 28$ & $118.6(7)$ \\
\hline $\mathrm{C} 38-\mathrm{C} 33-\mathrm{Sn} 2$ & $120.6(4)$ & $\mathrm{C} 27-\mathrm{C} 32-\mathrm{C} 31$ & $119.2(7)$ \\
\hline $\mathrm{C} 16-\mathrm{C} 15-\mathrm{C} 14$ & $120.4(6)$ & $\mathrm{C} 36-\mathrm{C} 35-\mathrm{C} 34$ & $120.5(7)$ \\
\hline $\mathrm{C} 17-\mathrm{C} 16-\mathrm{C} 15$ & $119.6(6)$ & $\mathrm{C} 30-\mathrm{C} 31-\mathrm{C} 32$ & $120.7(7)$ \\
\hline $\mathrm{C} 33-\mathrm{C} 34-\mathrm{C} 35$ & $120.7(6)$ & $\mathrm{C} 35-\mathrm{C} 36-\mathrm{C} 37$ & $120.0(7)$ \\
\hline $\mathrm{C} 6-\mathrm{C} 1-\mathrm{Sn} 1$ & $119.3(5)$ & $\mathrm{C} 31-\mathrm{C} 30-\mathrm{C} 29$ & $120.4(7)$ \\
\hline $\mathrm{C} 2-\mathrm{C} 1-\mathrm{Sn} 1$ & $121.4(5)$ & $\mathrm{C} 21-\mathrm{C} 22-\mathrm{C} 23$ & $120.7(6)$ \\
\hline $\mathrm{C} 2-\mathrm{C} 1-\mathrm{C} 6$ & $119.1(6)$ & $\mathrm{C} 2-\mathrm{C} 3-\mathrm{C} 4$ & $120.4(8)$ \\
\hline $\mathrm{C} 24-\mathrm{C} 25-\mathrm{C} 26$ & $118.9(6)$ & $\mathrm{C} 5-\mathrm{C} 4-\mathrm{C} 3$ & $119.2(7)$ \\
\hline
\end{tabular}

\section{TG/DCS curves}


$\mathrm{Ph}_{3} \mathrm{Sn} \_\mathrm{tHIMT} \_\mathrm{Cl}$

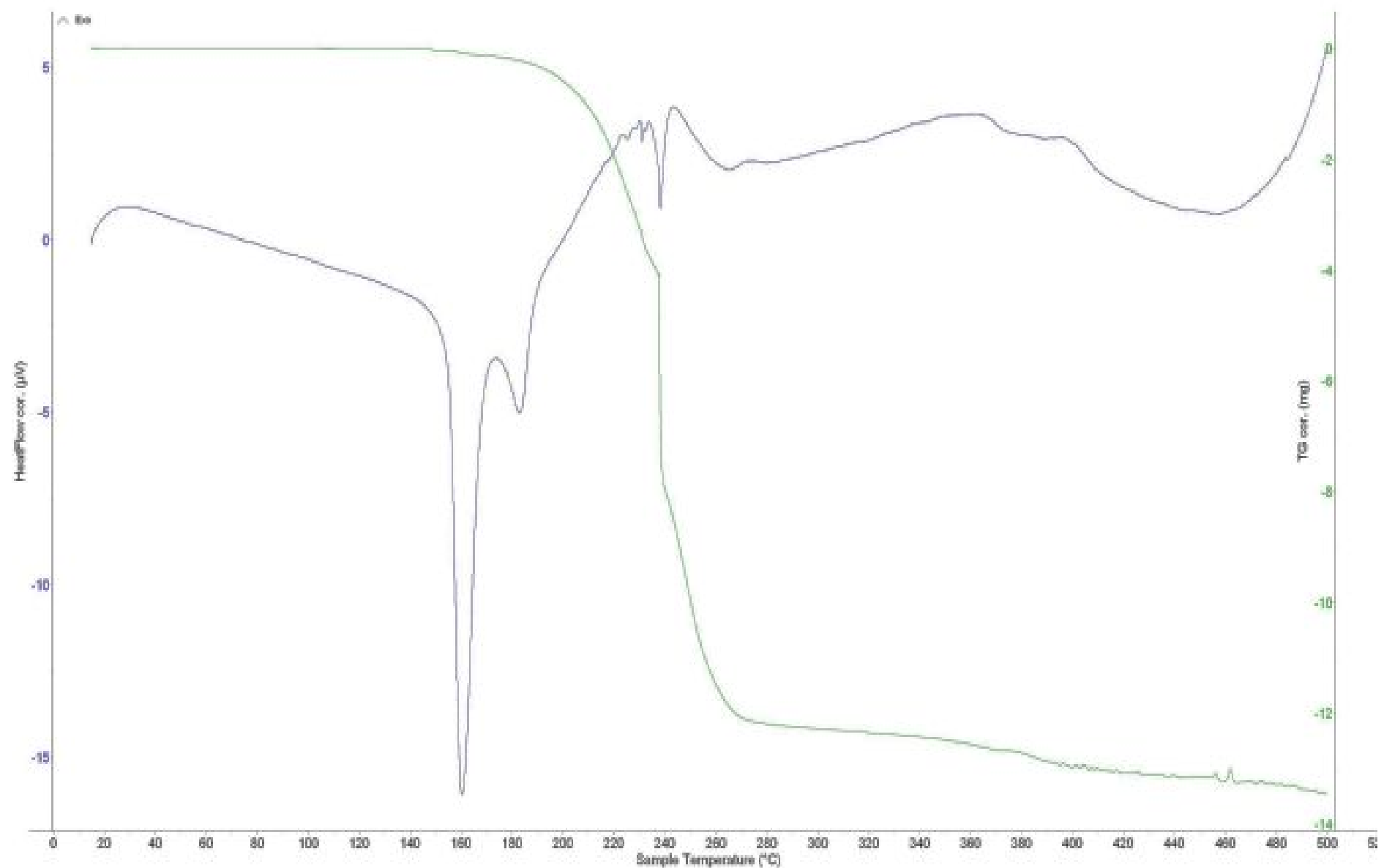

Figure S3.1 Superimposed TG-blue and DTA-green curves of complexes Ph $\mathbf{S h}_{3} \mathbf{S n}$ tHIMT_Cl (exo up) 
$\mathrm{Ph}_{3} \mathrm{Sn}$ _tHPMT_Cl (2)

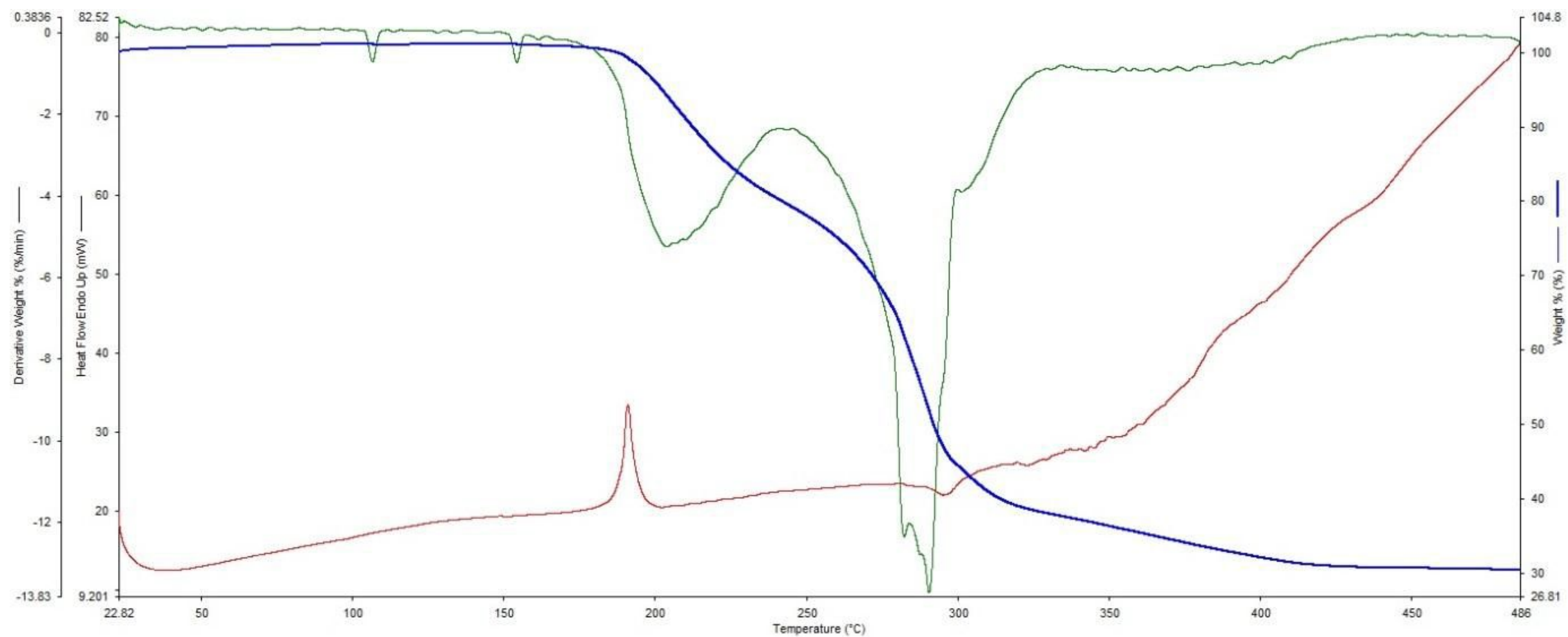

Figure S3.2 Superimposed TG-blue, DTG-green, DSC-red curves of complexes Ph3Sn_tHPMT_Cl (edno up), heating rate 10\%/min. 


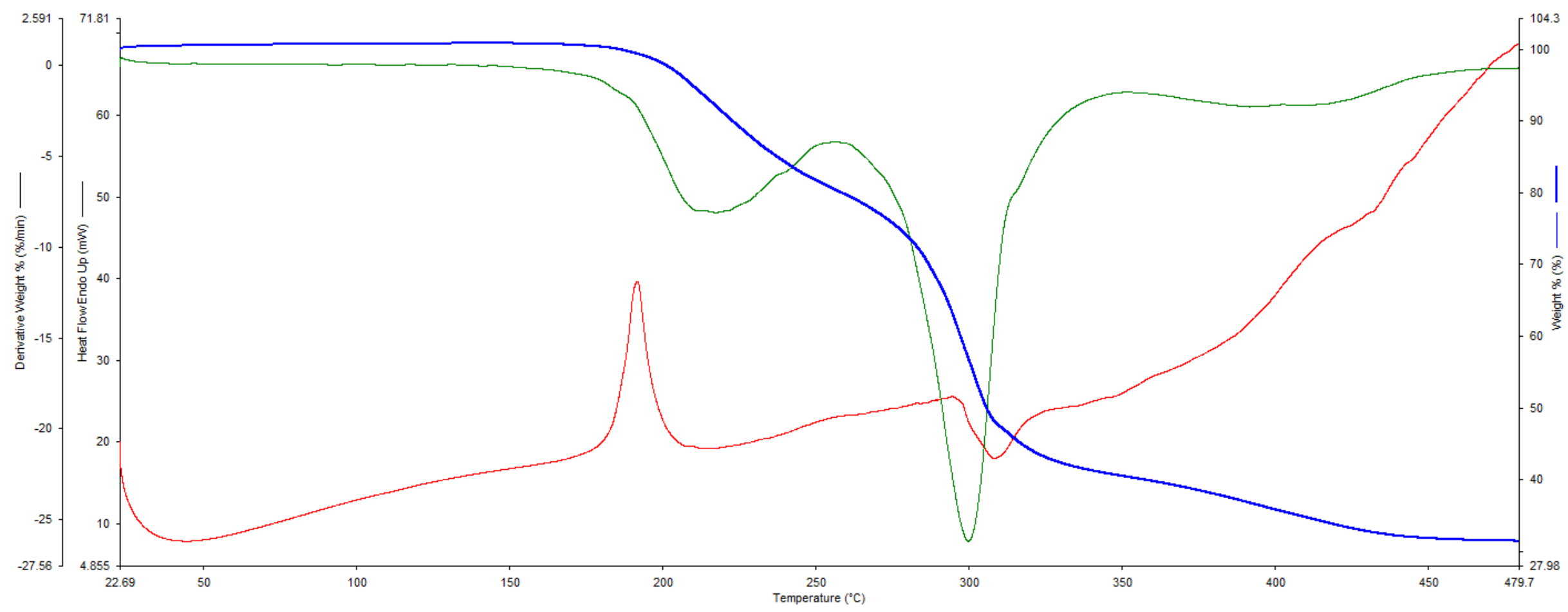

Figure S3.3 Superimposed TG-blue, DTG-green, DSC-red curves of complexes Ph3Sn_PMTtHIMT_Cl (edno up), heating rate 20\%/min. 
Ph 3 Sn_MIMT_Cl (3a)

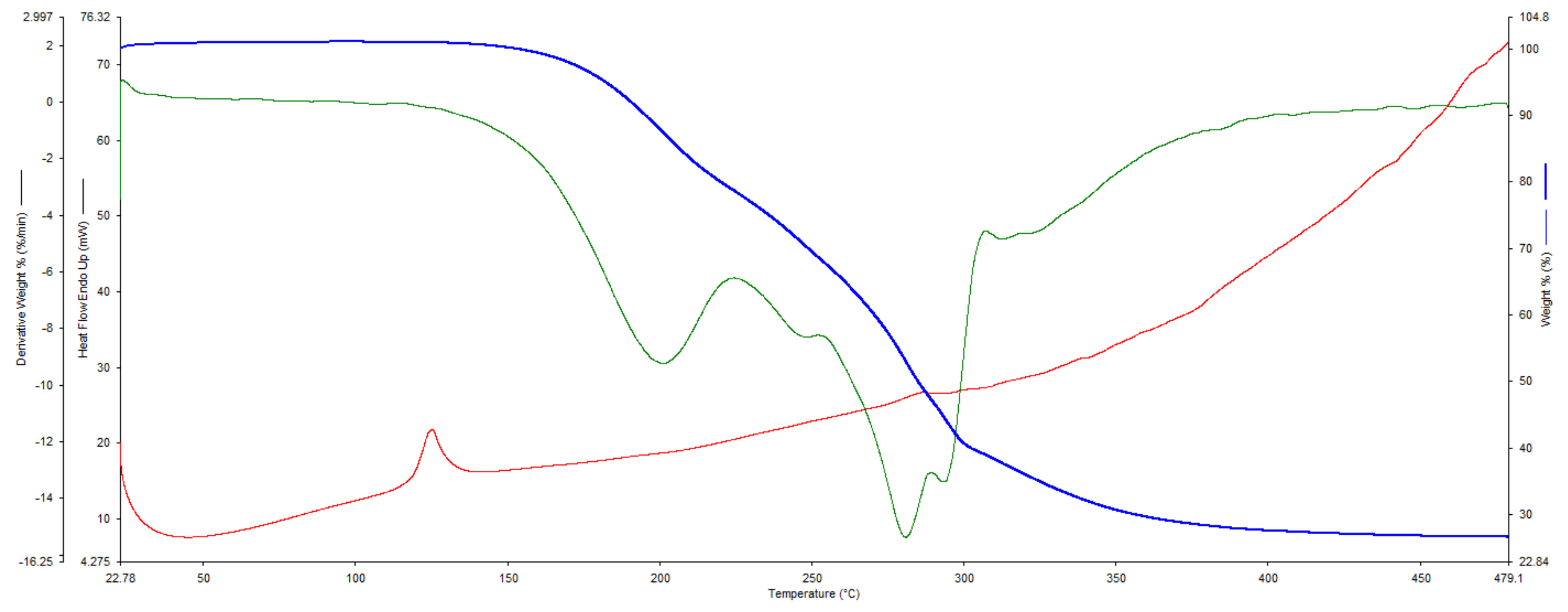

Figure S3.4 Superimposed TG-blue, DTG-green, DSC-red curves of complexes $\mathrm{Ph}_{3} \mathrm{Sn}_{-} \mathrm{MIMT}$ _Cl (3a) (edno up), heating rate 20\%/min. 


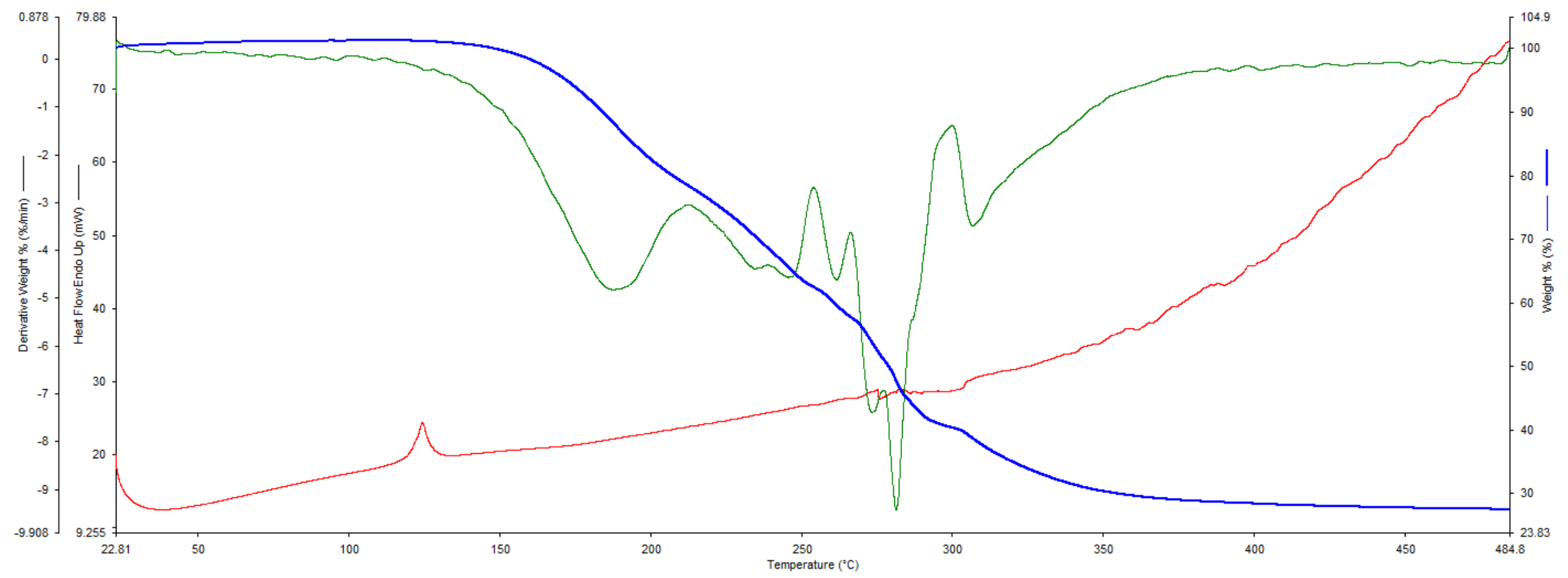

Figure S3.5 Superimposed TG-blue, DTG-green, DSC-red curves of complexes $\mathrm{Ph}_{3} \mathrm{Sn}$ _MIMT_Cl (3a) (edno up), heating rate $10^{\circ} / \mathrm{min}$. 


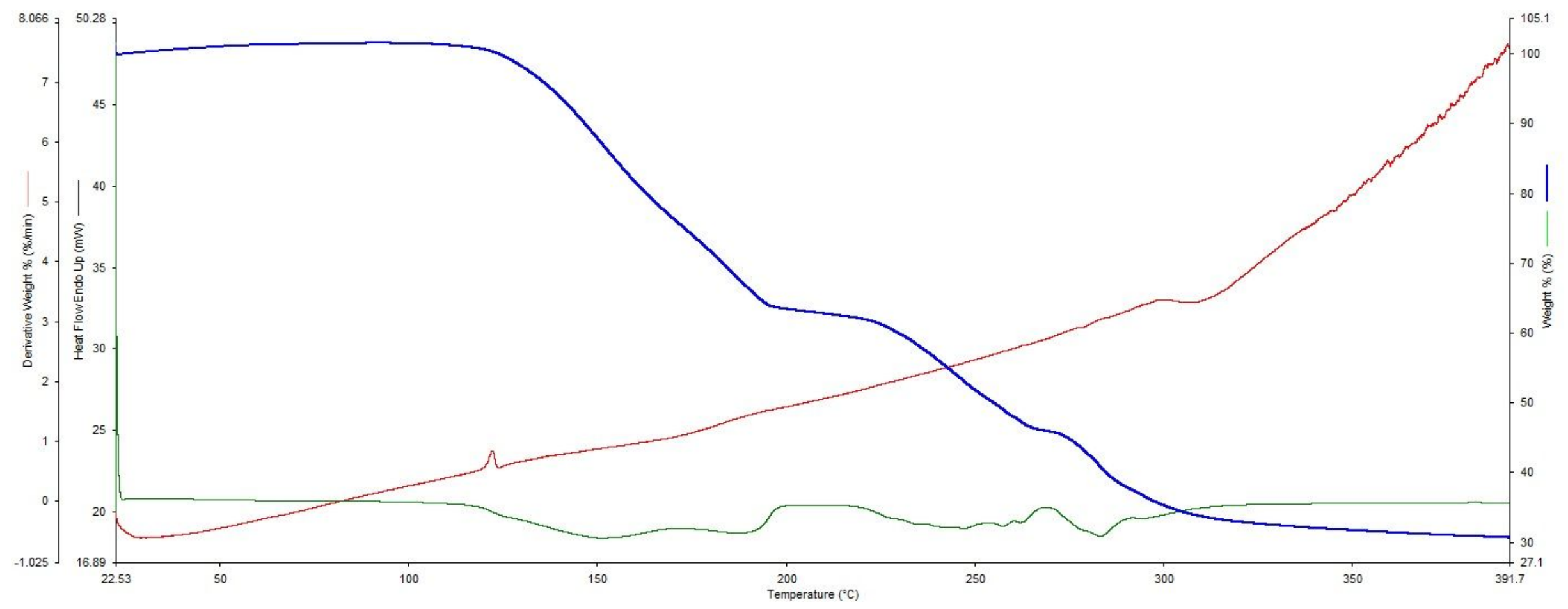

Figure S3.6 Superimposed TG-blue, DTG-green, DSC-red curves of complexes $\mathrm{Ph}_{3} \mathrm{Sn} \_\mathrm{MIMT} \_\mathrm{Cl}$ (3a) (edno up), heating rate $1 \%$ min. 
Ph ${ }_{3}$ Sn_MIMT_Cl (3b)

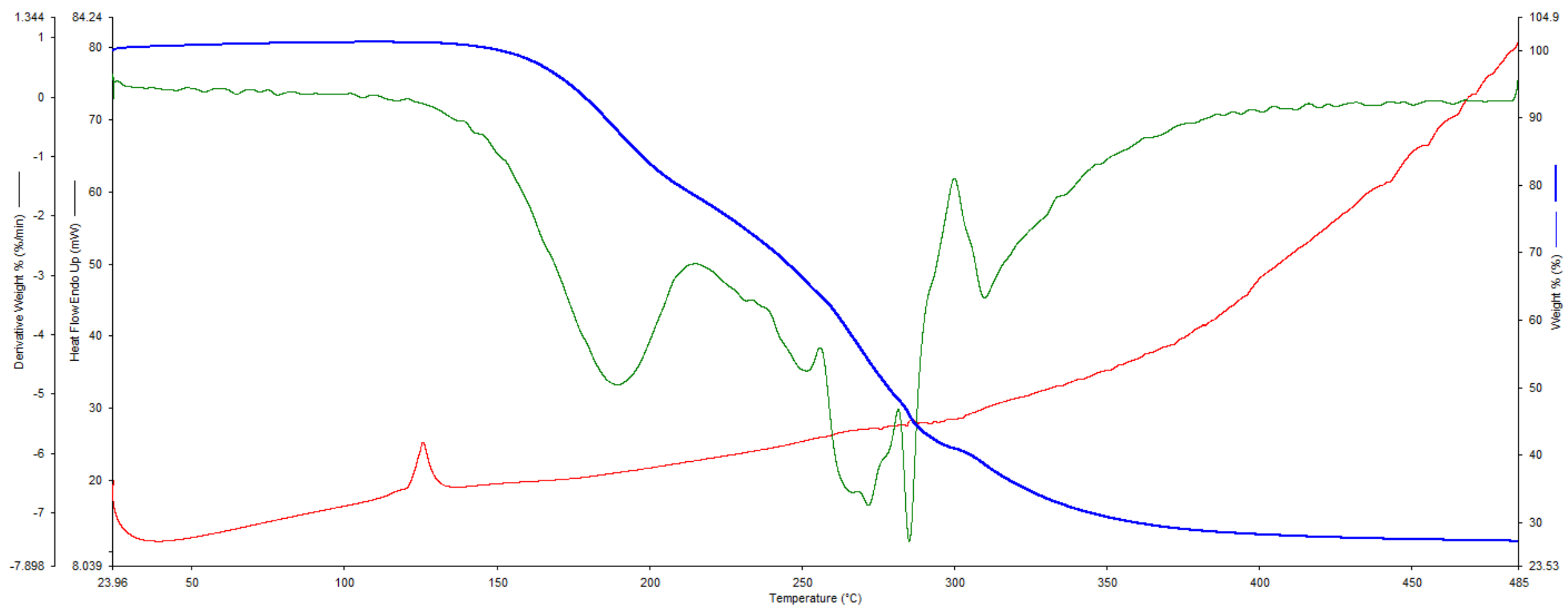

Figure S3.7 Superimposed TG-blue, DTG-green, DSC-red curves of complexes $\mathrm{Ph}_{3} \mathrm{Sn} \_\mathrm{MIMT}$ Cl (3b) (edno up), heating rate 20\%/min 
$\mathrm{Ph}_{3} \mathrm{Sn} \_$DMF_Cl (5)

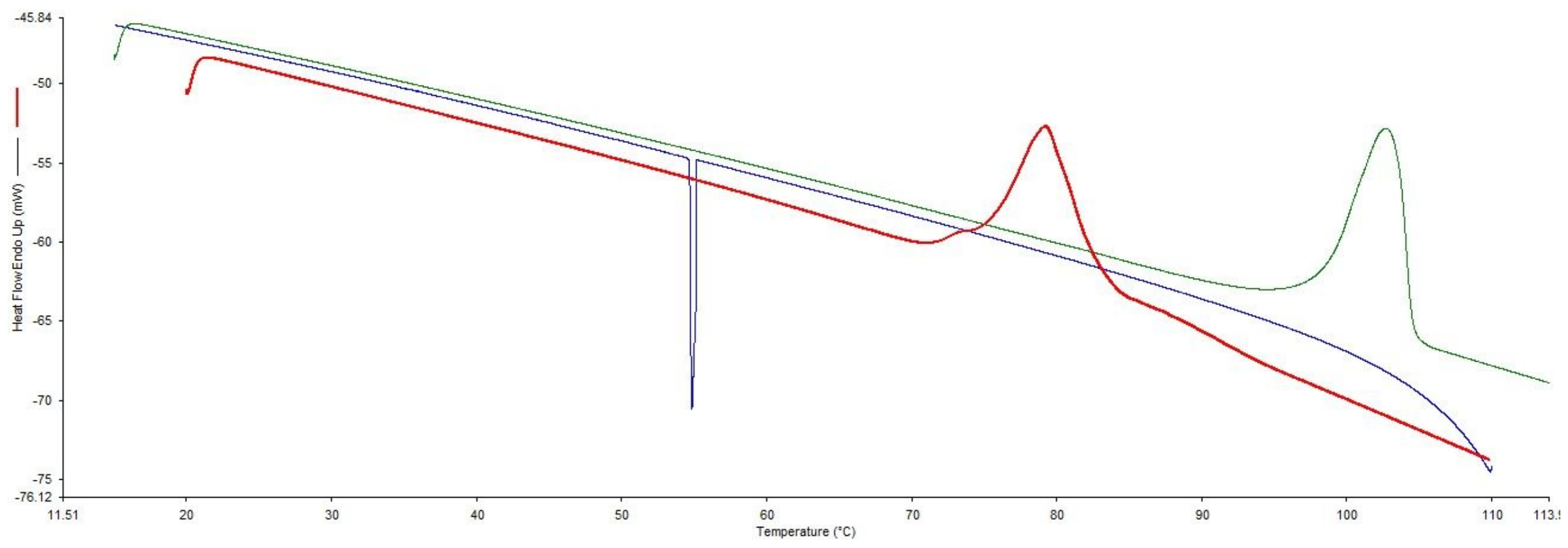

Figure S3.8 Superimposed DSC curves of $\mathrm{Ph}_{3} \mathrm{Sn}_{-} \mathrm{DMF} \_\mathrm{Cl}$ (5), red- first heating , blue-cooling, green-second heating (edno up). 
$\mathrm{Ph}_{3} \mathrm{Sn}$ DDMSO_Cl (6a)

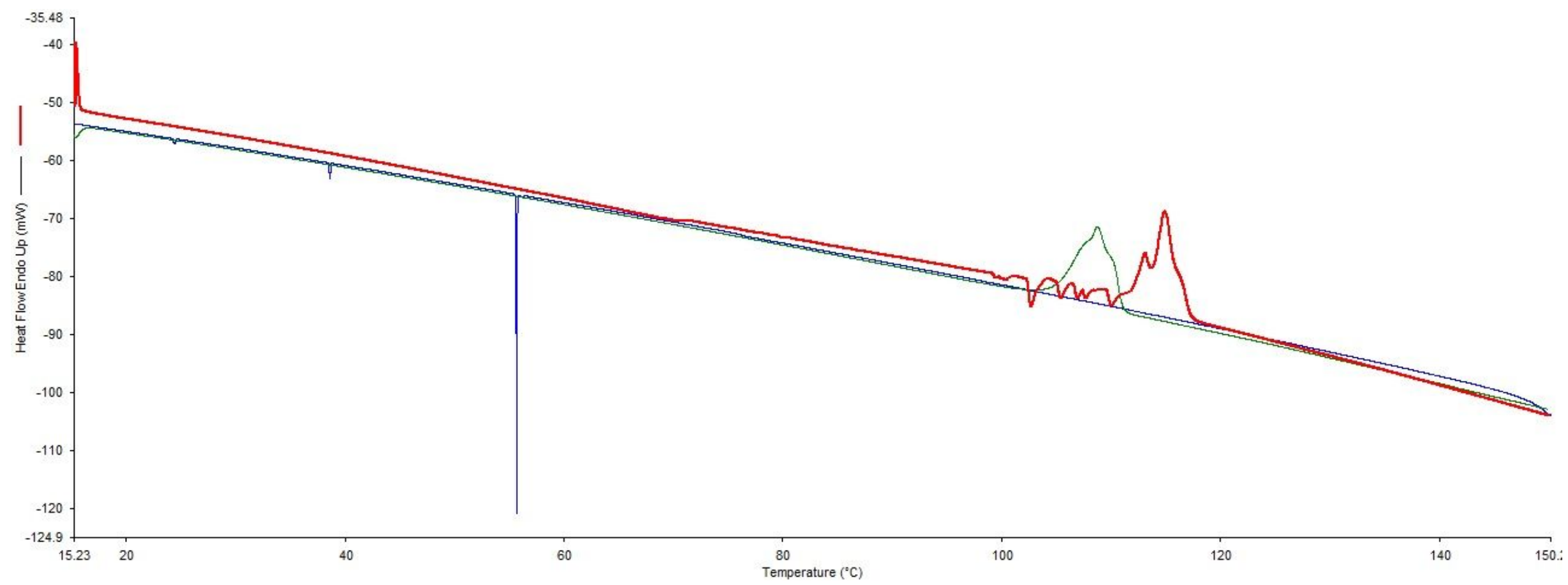

Figure S3.9 Superimposed differential scanning calorimetric curves of Ph ${ }_{3} \mathrm{Sn}_{\text {_DMSO_Cl }}$ (6a), red- first heating , blue-cooling, green-second heating (edno up). 
4. Powder X-Ray diffractograms



Figure S4.1 Comparison of theoretical and experimental powder pattern of $3 a$ 


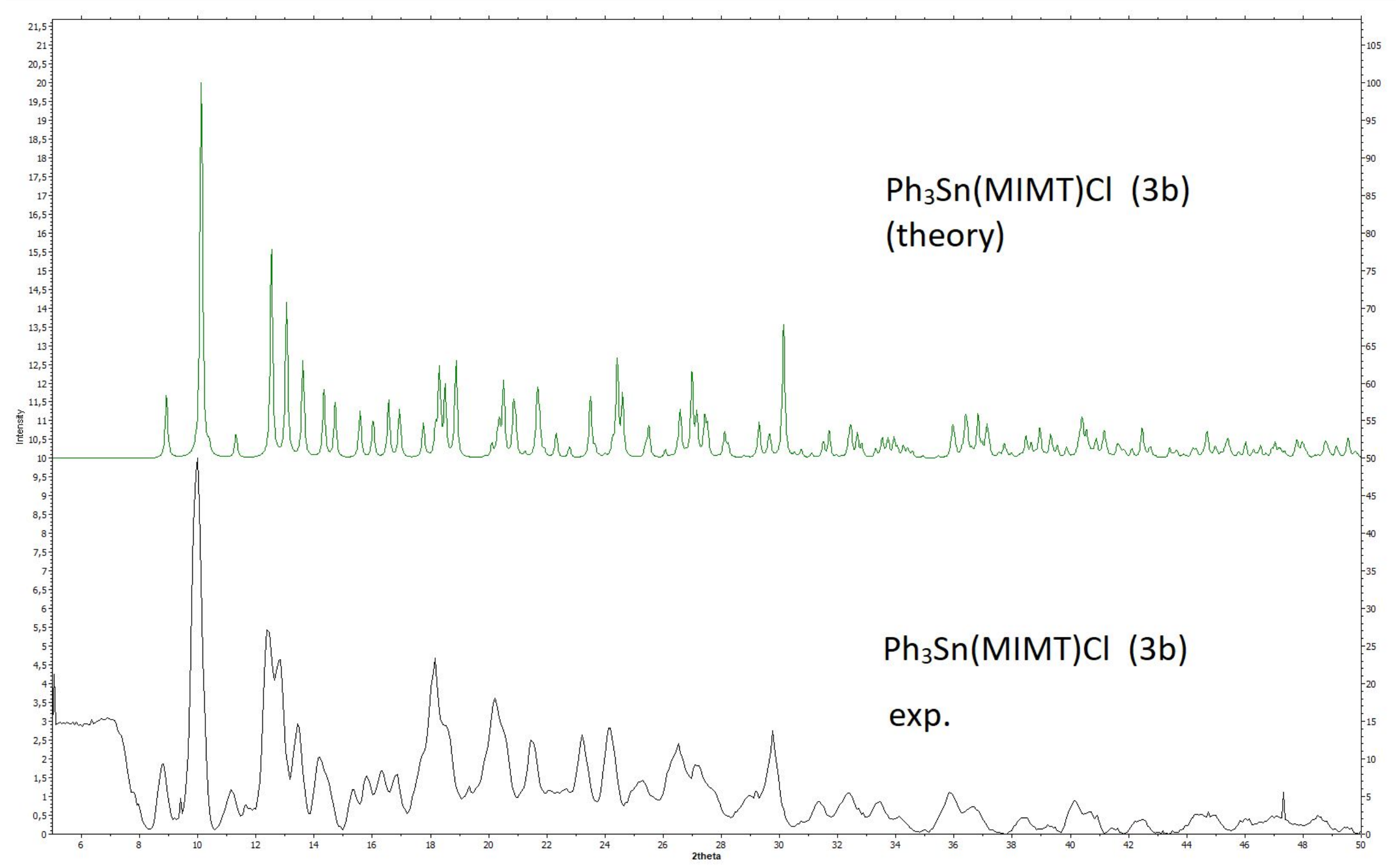

Figure S4.2 Comparison of theoretical and experimental powder pattern of $\mathbf{3 b}$ 


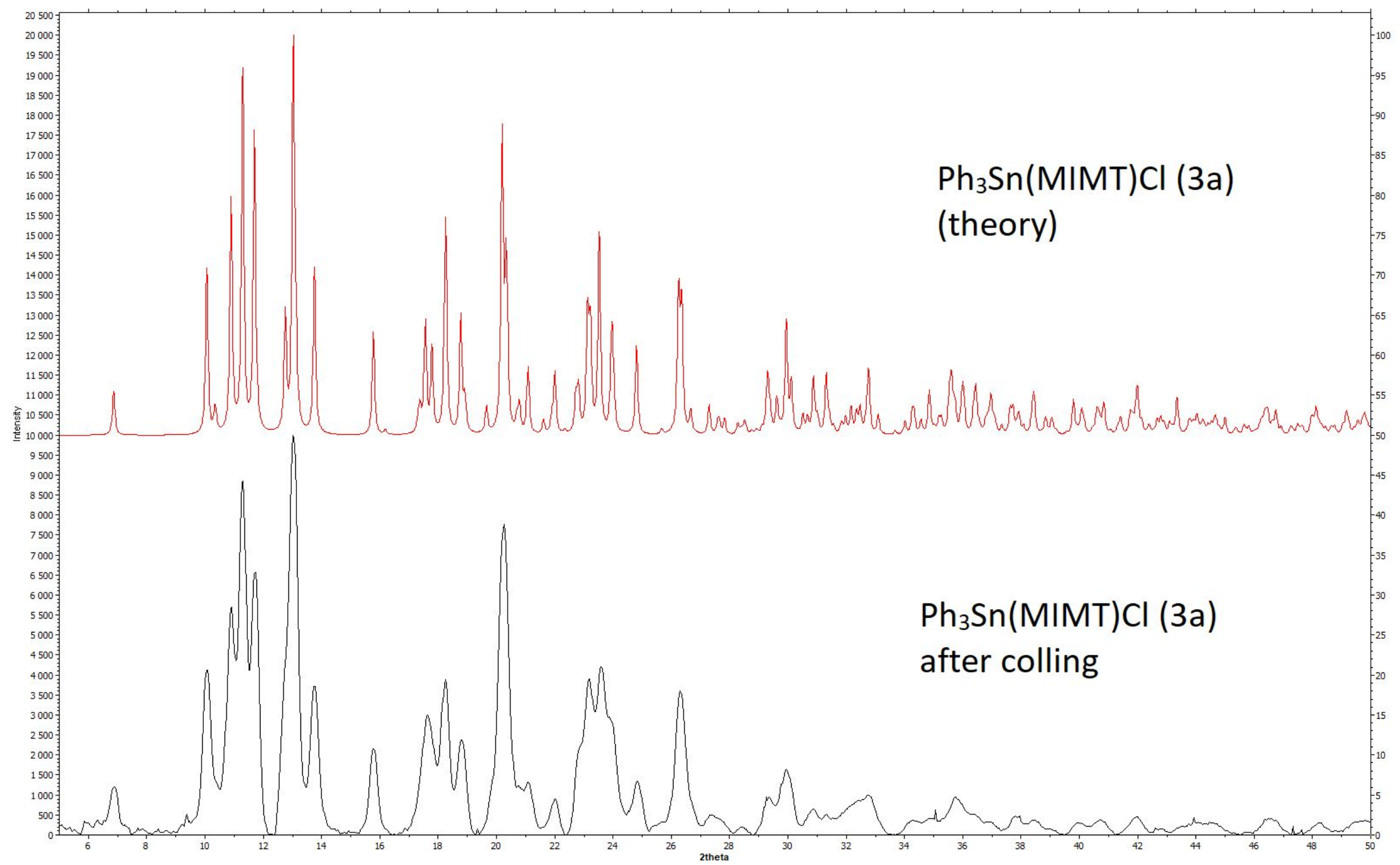

Figure S4.3 Comparison of powder pattern of 3a after cooling with theoretical one 


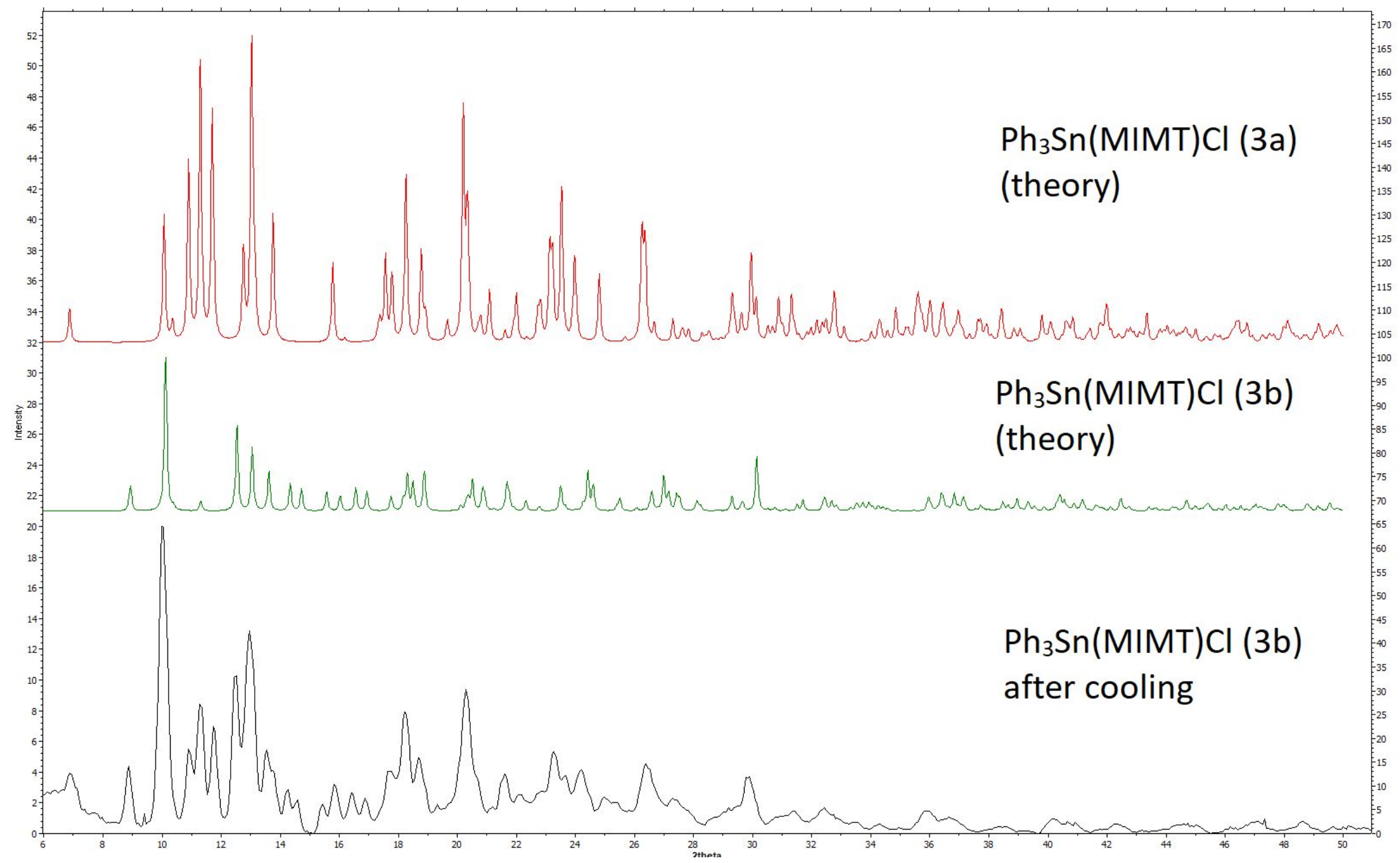

Figure S4.4 Comparison of powder pattern of $\mathbf{3 b}$ after cooling with theoretical one 


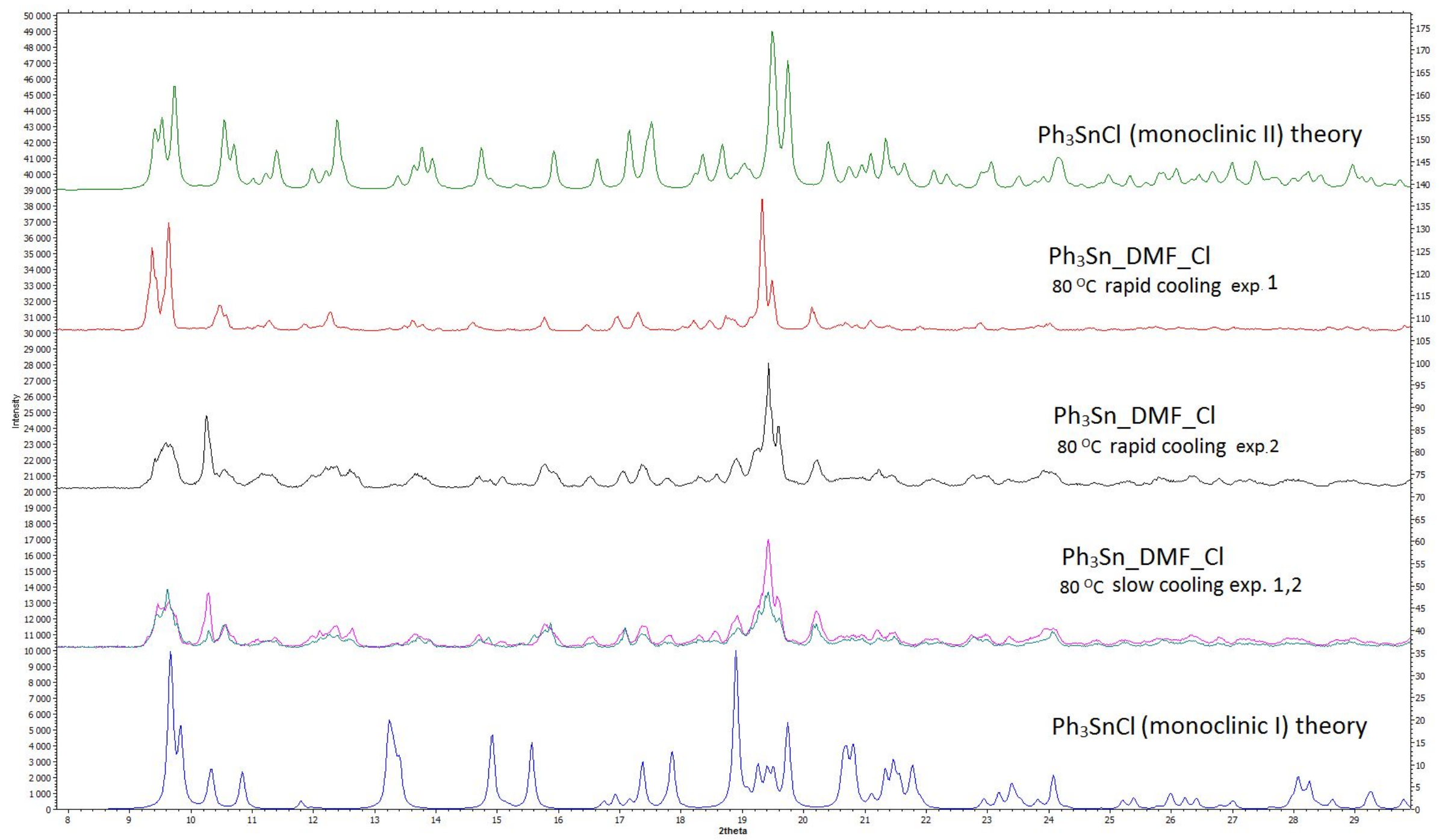

Figure S4.5 Comparison of powder pattern of 5 heated to $80^{\circ} \mathrm{C}$ and cooled with powder pattern of substrates 


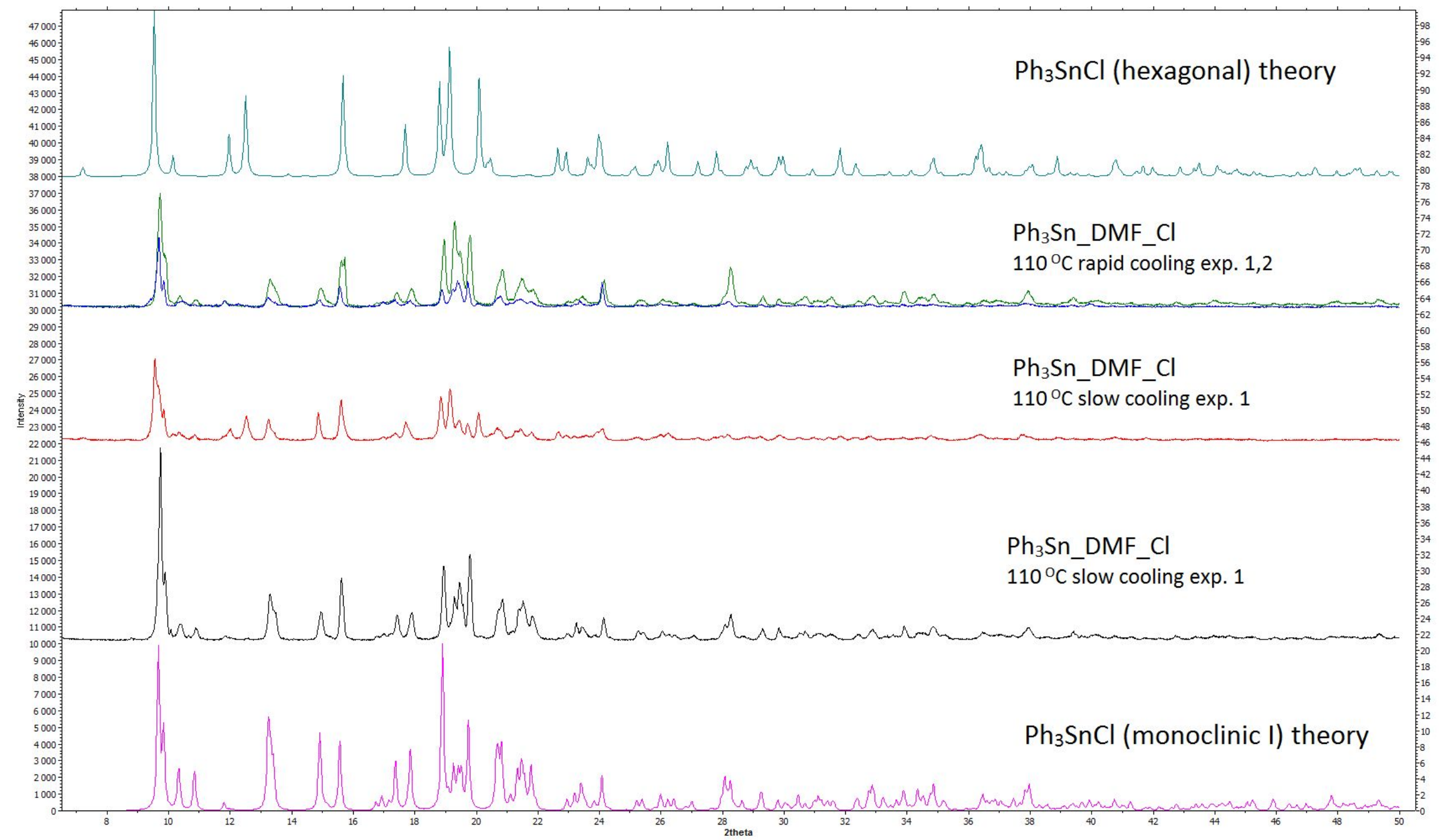

Figure S4.6 Figure S4.5 Comparison of powder pattern of 5 heated to $110^{\circ} \mathrm{C}$ and cooled with powder pattern of substrates 


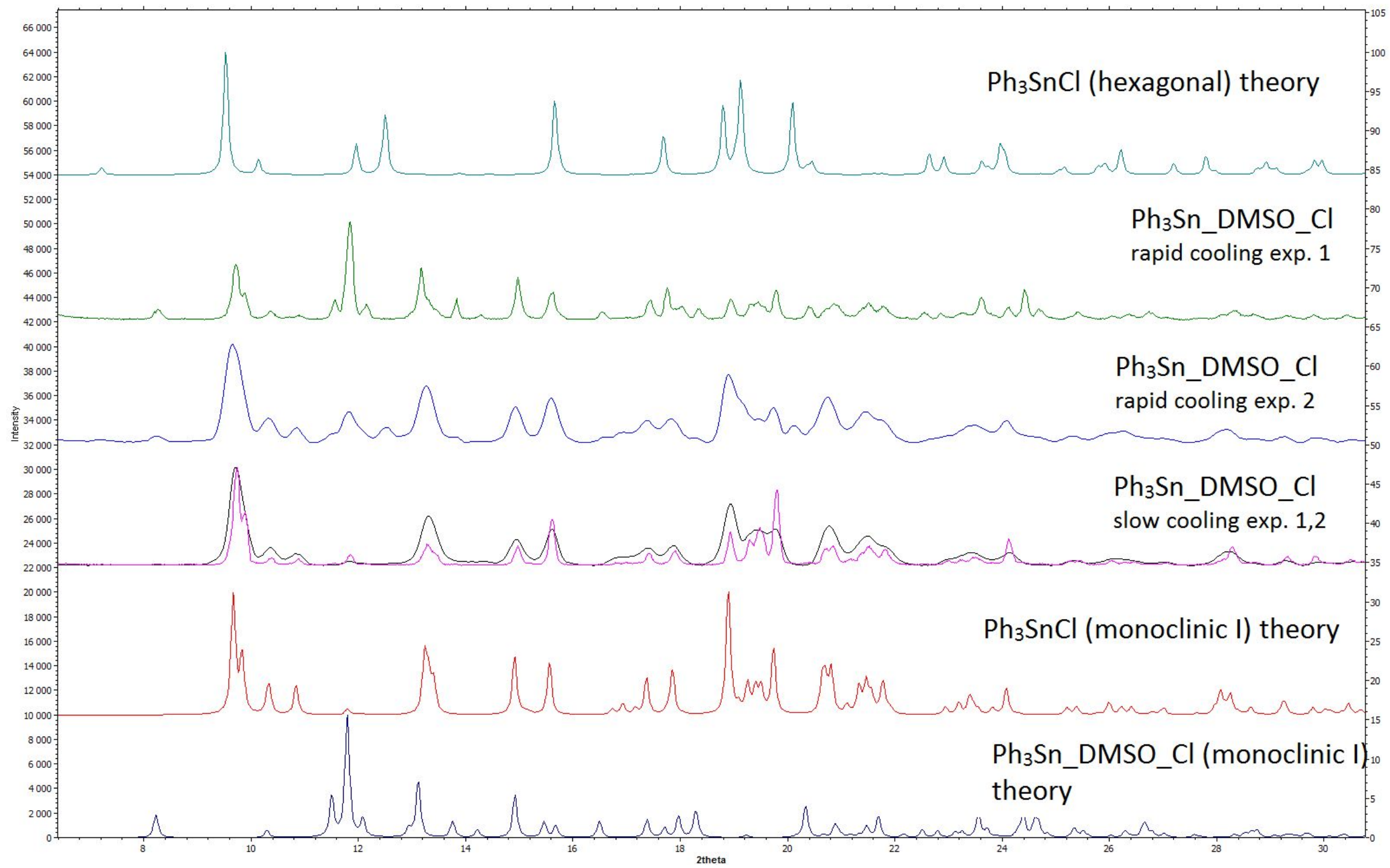

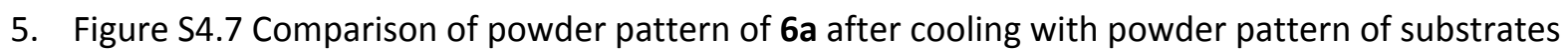




\section{Movies}

Movie 1 - real time

Movie 2- 64x speeded up along the entire length of the film

Movie $3 a-64 x$ speeded up till the $13 \mathrm{sec}$, than real time

Movie3b- $8 \mathrm{x}$ speeded up along the entire length of the film

Movie 4- 16x speeded up till the 20sec, than real time

Movie 5- real time

\section{Hirshfeld fingerprints}

Table S3. Hirshfeld fingerprints of studied compounds

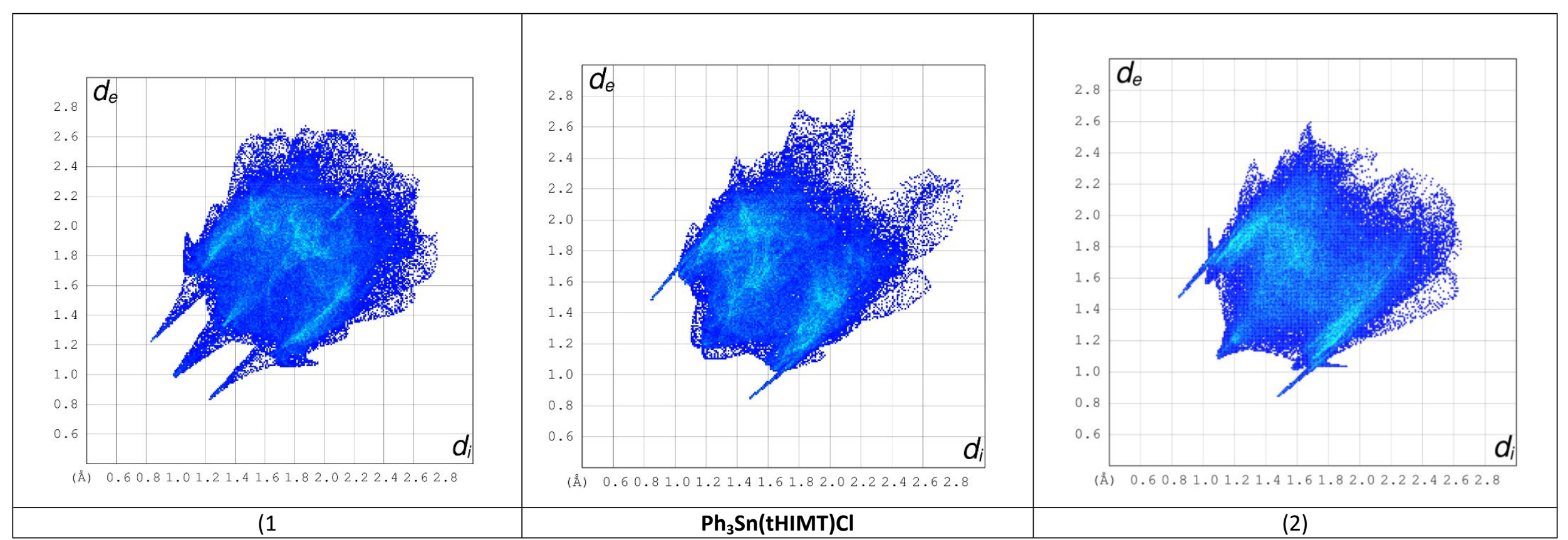




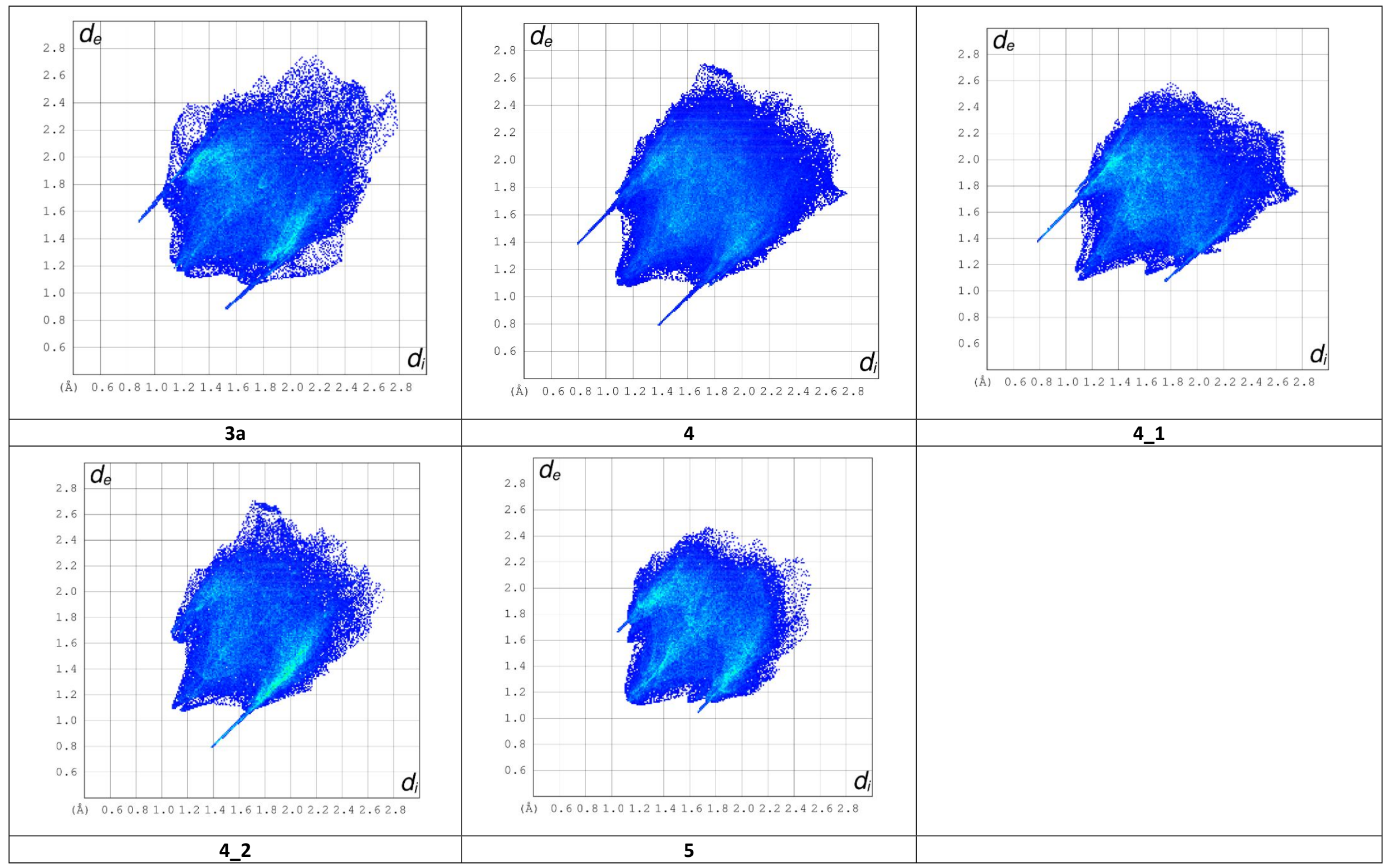




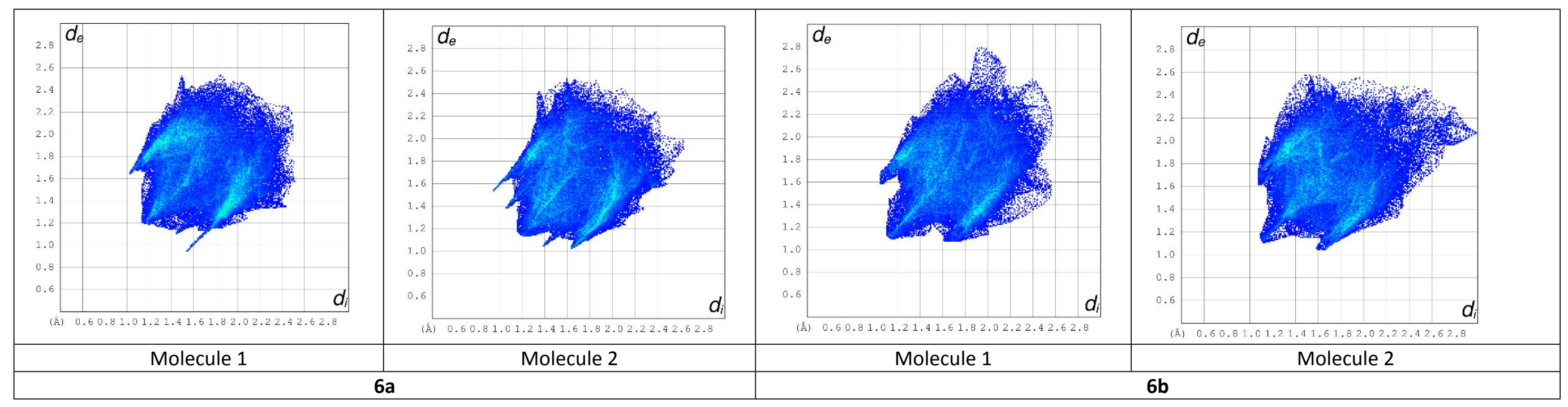

